\title{
A Study on the Dynamic Performance for Hydraulically Damped Rubber Bushings with Multiple Inertia Tracks and Orifices: Parameter Identification and Modeling
}

\author{
Chao-Feng Yang, ${ }^{1}$ Zhi-Hong Yin, ${ }^{1}$ Wen-Bin Shangguan, ${ }^{1,2}$ and Xiao-Cheng Duan ${ }^{1,2}$ \\ ${ }^{1}$ School of Mechanical \& Automotive Engineering, South China University of Technology, Guangzhou 510641, China \\ ${ }^{2}$ Ningbo Tuopu Group Co., Ltd., Ningbo 315800, China \\ Correspondence should be addressed to Zhi-Hong Yin; mezhyin@scut.edu.cn
}

Received 21 October 2015; Accepted 26 November 2015

Academic Editor: Radoslaw Zimroz

Copyright (C) 2016 Chao-Feng Yang et al. This is an open access article distributed under the Creative Commons Attribution License, which permits unrestricted use, distribution, and reproduction in any medium, provided the original work is properly cited.

\begin{abstract}
Hydraulically damped rubber bushings (HDBs) are important for vehicle noise, vibration, and harshness (NVH) performance as they are able to decay the vehicle's oscillation induced by engine and road. The dynamic stiffness and loss angle of an HDB are crucial and it is significant to investigate the relations between the design parameters with the dynamic stiffness and loss angle. Therefore, the force-deflection relation of the HDB is measured statically and the dynamic stiffness and loss angle are measured dynamically and the test data are analyzed with a view to examine how the measurement results are influenced by the design parameters (the number of the fluid tracks). Compared with the results predicted by a nonlinear lumped parameter model whose parameters are extracted by a parameter identification technique, using the model, the effect of the main rubber and the fluid track on the dynamic stiffness and the loss angle is investigated. A unified analytical model of HDB is also developed with the purpose of predicting the static and dynamic characteristics, and the predictions are shown to be well correlated with the measurement data. The good correlation suggests the validity of the model and the parameter identification implementation.
\end{abstract}

\section{Introduction}

Motivation. Rubber bushings are commonly used in engine subsystems, vehicle body, and vehicle suspension to damp their oscillations excited by dynamic loads, thus improving driving safety, ride comfort, and handling performances [19]. Compared with conventional rubber bushings, an hydraulically damped rubber bushing (HDB) can provide a high viscous damping coefficient in certain frequency range, while exhibiting amplitude dependent properties and frequency dependent properties [5-9], which is one of the advantages of an HDB. In designing the frequency characteristics of an $\mathrm{HDB}$, designers take care of the value and peak frequency of the loss angle of the HDB mostly, which are dependent upon the performance of the main rubber spring, the fluid property, the shapes of two chambers, the fluid tracks, and so forth. Some effective methods and models have to be invited to calculate the influences of those factors on the dynamic characteristics of an HDB during the design stage. The dynamic performances of an HDB are often characterized with two terms, dynamic stiffness and loss angle, which are defined as the amplitude ratio of the load response to the displacement response and the phase difference between the load and displacement responses in frequency domain, respectively [4-10].

The lumped parameter (LP) model [10] is often employed to estimate the dynamic performances of an $\operatorname{HDB}[1,2,4-$ 9]. Each lumped parameter represents a certain physical meaning, and the dynamic characteristics of the HDB can be described as a function of lumped parameters explicitly [9, 10]. However, the traditional method of parameter identification is a high-cost and time-consuming task for some lumped parameters, such as the HDB's effective piston area and the compliances of two fluid chambers. To obtain these design 
variables of an $\mathrm{HDB}$, a new parameter identification method is proposed based on the characteristic frequency points of the dynamic performances of HDB in this paper.

During the design stage of an HDB, it is not easy to adjust the maximum frequency of loss angle to the same frequency of the most disruptive excitations with one fluid element due to geometry limitations of the fluid track [11]. Besides, the hydraulic damping mechanism is relatively sensitive to the operating environment while multiple damped dynamic vibration absorbers have good robustness against it [12]. However, the influences of fluid track number on the dynamic characteristics of an HDB with multiple inertia tracks and orifices as well as its practical application issues are not fully investigated.

Therefore, the motivations of this paper are to develop a superior parameter identification method for the HDB and to reveal the influences of the geometry size and the number of fluid tracks on the dynamic characteristics of the HDB by test and simulation approaches.

Literature Review. The first HDB was introduced in the mid1980s on MacPherson-type front suspension systems [11]. Similar designs are already used as HEM and are adapted for application with cylindrical sleeve-type bushing joints simply $[11,13]$. There are also many patents [14-20] that claim the dynamic characteristics features of some specific HDBs in frequency domain; however, no analytical analysis or even measured dynamic properties are provided [4-8]. Further, very limited articles have reported the dynamic properties characterization and modeling issues of HDB.

$\mathrm{Lu}$ and Ari-Gur $[1,2]$ present a linear LP model for an HDB with two inertia tracks and derived the natural frequency analytically. Then, the model is applied to the simulation of an HDB with multiple inertia tracks. However, their model is not validated experimentally. Sauer and Guy [3] present a fluid system model for an HDB with a bypass track and one inertia track in parallel, which serves as a relief valve securing the HDB and the adjacent structure from undesirable shocks, but the detailed calculation conclusions are not carried out. Shangguan and $\mathrm{Xu}$ [9] propose a nonlinear lumped parameter state-space model (by introducing a nonlinear track resistance $R_{i}$ ) for an HDB with one inertia track, and the model is verified by experiments, while the analytical study is conducted based on the linearized model. Sevensson and Hakansson [21] and Pan et al. [22] provide an empirical mathematic model including a nonlinear spring with several fluid elements and elastoplastic elements coupled in parallel, but the calculation results show that the accuracy of the model is not satisfactory for low amplitude excitation. Arzanpour and Golnaraghi $[23,24]$ present a linear LP model of an HEM employing the same approach as that for an HDB with one inertia track. Chai et al. [4-8] initiate a laboratory device with alternative internal configurations, and the dynamic characteristics for different configurations are investigated comparatively, as well as the steady state and transient responses of the device. However, the influence of the number of the fluid tracks on the dynamic characteristics is not discussed. The parameters of LP models of HDB with multiple inertia tracks are identified by methods of
TABLE 1: Geometric and material parameters of a typical HDB.

\begin{tabular}{lc}
\hline Parameter & Value \\
\hline Length of inertia track $l_{i} / \mathrm{m}$ & 0.19 \\
Cross-sectional area of the track $A_{o} / A_{i} / \mathrm{m}^{2}$ & $9.83 \times 10^{-6}$ \\
Wet perimeter of the inertia track $\chi_{i} / \mathrm{m}$ & $1.27 \times 10^{-2}$ \\
Hydraulic diameter of the inertia track $d_{H} / \mathrm{m}$ & $3.087 \times 10^{-3}$ \\
Length of the orifice $l_{o} / \mathrm{m}$ & 0.012 \\
Density of the fluid $\rho /\left(\mathrm{kg} / \mathrm{m}^{3}\right)$ & 1112.80 \\
Poisson ratio of the rubber spring $\nu$ & 0.50 \\
Density of the rubber spring $\rho_{r} /\left(\mathrm{kg} / \mathrm{m}^{3}\right)$ & 1050.00 \\
\hline
\end{tabular}

curve fitting and Nyquist diagrams $[4,8]$. However, the phenomenon of the excitation amplitude-invariant fixed points on the dynamic performance curves of HDBs with different internal configurations is not investigated.

In summary, no uniform LP models have been developed to analyze the influences of the number of long fluid tracks and orifices on the dynamic characteristics of HDBs. A thorough understanding of the mechanism of HDBs is needed.

Scope and Objectives. As a passive vibration isolation component in the vehicle suspension and steering wheel assemblies, HDBs play an important role in dealing with vehicle system vibration issues, such as brake judder and shimmy $[3,11]$. The configuration of a typical HDB and the runner plate are illustrated in Figures 1 and 2, respectively. Its geometric and material parameters are listed in Table 1.

The elastomeric rubber spring, enclosed by inner and outer metal sleeves, has two functions: to support the static load of the vehicle and to provide partial static stiffness and somewhat damping of the suspension system. The typical passive hydromechanical bushing usually consists of two almost same fluid chambers connected by one or more inertia tracks and/or orifices. Typically, the fluid chambers filled with an antifreeze and water mixture which has appropriate dynamic and thermal properties required for isolation and control [25]. In the event of a dynamically oscillatory excitation, the chamber pressures change in radical direction and the volume compensation will take place between two fluid chambers. Thus, the fluid flows back and forth through the fluid tracks as an oscillating mass to provide hydraulic damping $[4-9,11,13]$.

Based on the above analysis, HDB's engineering designs are conceptually identical to those of HEM, but their structures, working mechanisms, and dynamic properties are essentially different [4-8]. In particular, the lower chamber of the HEM is very thin, and the pressure in the lower chamber is usually smaller than that in the upper chamber. On the contrary, the two chamber pressures of the HDB are relatively high. Therefore, the LP models developed for HEM cannot be directly applied to HDB [4-8].

The disadvantage of the HDB with inertia track is that the flow velocity of the liquid column in the inertia track becomes very slow due to the viscosity of the fluid once the frequency excitation reaches its resonant frequency; that is, it usually can be assumed that the flow "shut off" [25-28], 


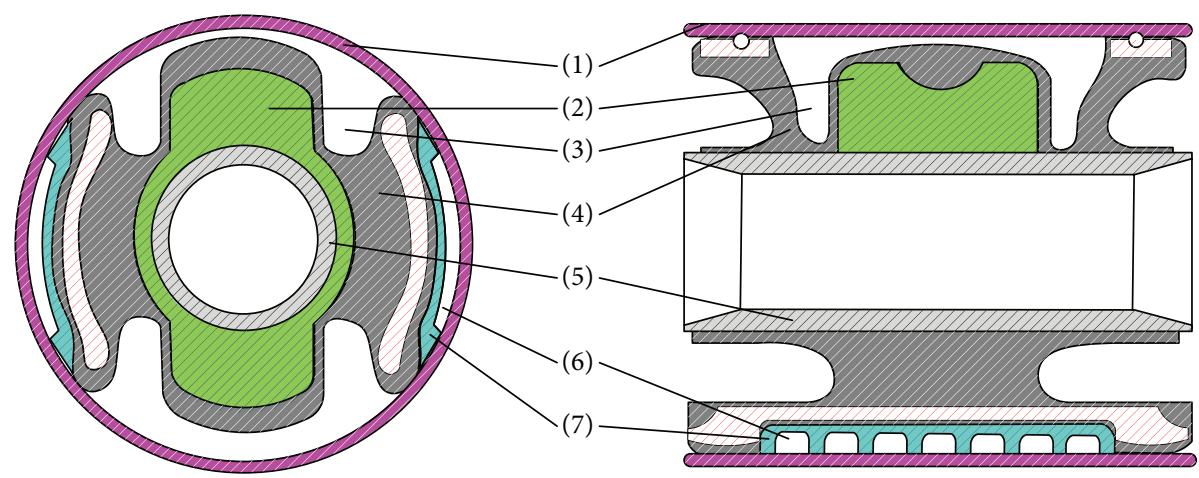
(1) Outer metal sleeve
(5) Inter metal sleeve
(2) Stopper member
(6) Inertia track
(3) Fluid chamber
(7) Fluid track plate

(4) Rubber spring

Figure 1: The cross-sectional view of HDB.

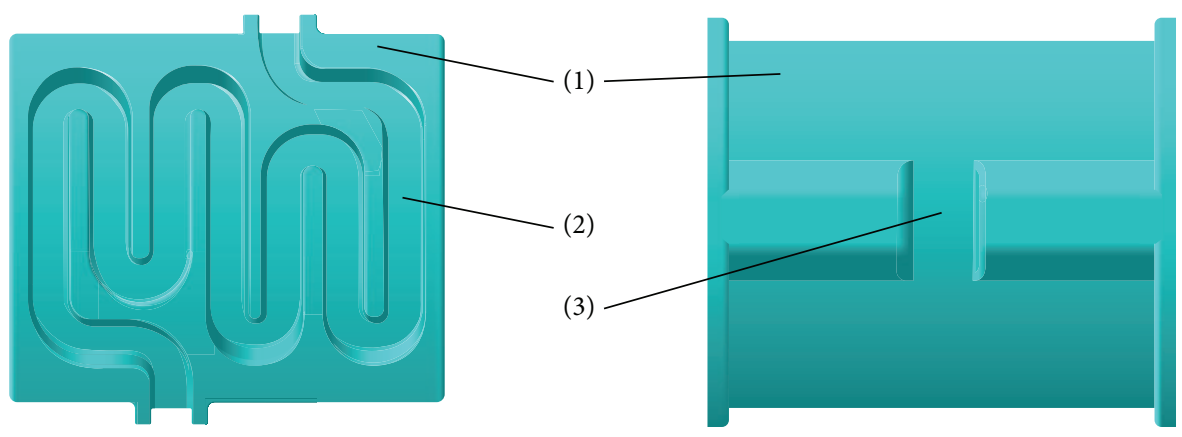

(1) Fluid track plate

(2) Inertia fluid track

(3) Orifice fluid track

FIGURE 2: The fluid track plate of hydraulic bushing.

which is undesirable. However, the HDB with large orifice can provide sufficient damping in a wide frequency range, while the damping in the low frequency range is deteriorated [11].

The fluid tracks plates of HDBs usually have irregular geometry and vary from sample to sample significantly. In this paper, the HDBs with different fluid track plates are manufactured and studied, which can provide insights into the features needed for scientific verification.

The objectives of this paper include the following: (a) design a controlled experiment to test the static and dynamic performances of HDB with multiple inertia tracks and orifices by change of the fluid track plate and investigate the excitation amplitude-invariant fixed points on the dynamic characteristics of HDBs with different fluid track plates; (b) present a new parameter identification technique and develop a nonlinear unified LP model for the HDB with multiple inertia tracks and orifices to analyze the influences of the number of different tracks on the dynamic characteristics of the HDB and estimate the peak frequency of the loss angle; (c) validate the unified analytical model by comparing simulation results with test data and predict the influences of the number of fluid tracks on the dynamic performances of the HDB.

\section{Design of HDB with Multiple Configurations and Experimental Study}

2.1. Types and Experimental Procedure. A group of HDB types with varied configurations are manufactured to investigate the influences of the inertia tracks and orifices on the HDB performances.

The basic features of the 10 tested HDBs are summarized in Table 2. For each type (HB1-HB5), two identical samples are prepared. Within these HBDs, the type of HB6 is special because it denotes the main rubber of the HDB.

Because the fluid of $\mathrm{HB} 1$ and $\mathrm{HB} 3$ does not provide hydraulic damping, the LP models are not considered in this study. Different LP models for those HDBs (HB1, HB2, HB4, and HB5) are illustrated in Figure 3. $K_{r}$ is the complex stiffness of the main rubber and $B_{r}$ is the damping ratio of the main rubber. $C_{1}$ and $C_{2}$ are the volumetric compliances of the two fluid chambers induced by the rubber spring and $K_{1}$ and $K_{2}$ are the volumetric stiffness of the two fluid chambers, respectively. $A_{p 1}$ and $A_{p 2}$ represent the effective pumping areas of the upper and the lower chambers, assuming that the behavior of the rubber spring can be approximated by 
TABLE 2: The configurations of HDB samples.

\begin{tabular}{lll}
\hline Types & Configurations & Structural features \\
\hline HB1 & HDB with one inertia track & Block one inertia track \\
HB2 & HDB with two inertia tracks & Install two fluid track plates symmetrically (one inertia track on each plate) \\
HB3 & HDB with no track & Block two inertia tracks \\
HB4 & HDB with one inertia and one orifice track & $\begin{array}{l}\text { Install two fluid track plates symmetrically (one plate with an inertia track } \\
\text { and the other plate with an orifice) }\end{array}$ \\
HB5 & HDB with two orifice tracks & Install two fluid track plates symmetrically (both with an orifice) \\
HB6 & Main rubber & Empty the chambers of the HDB with multiple configurations \\
\hline
\end{tabular}

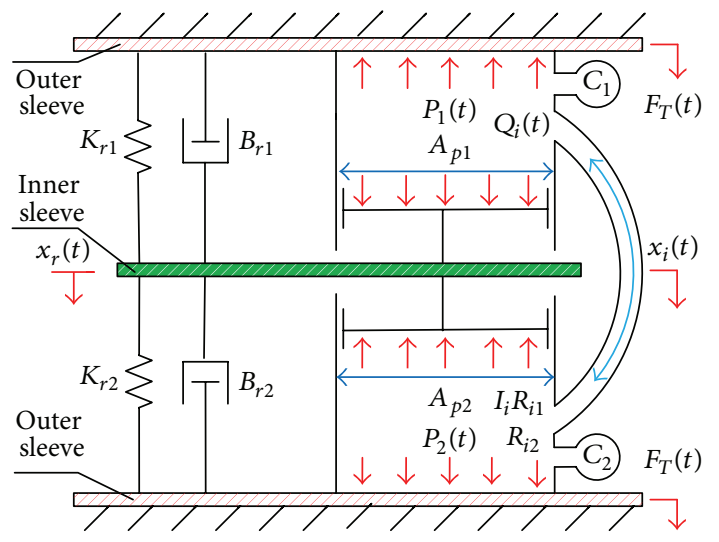

(a)

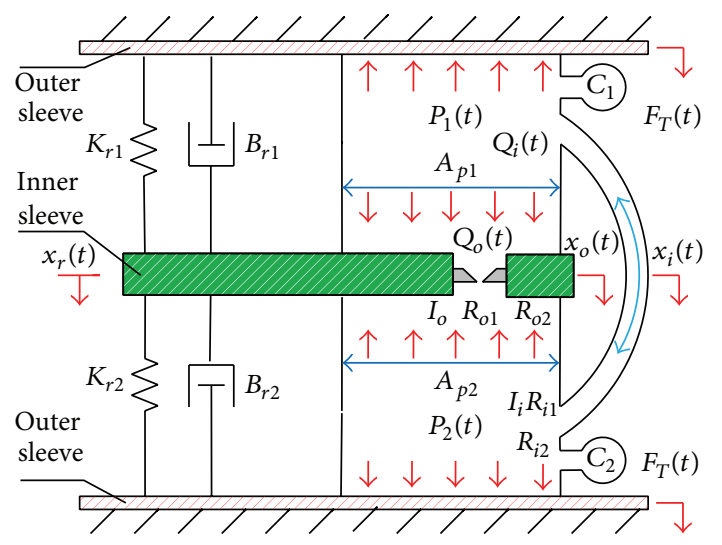

(c)

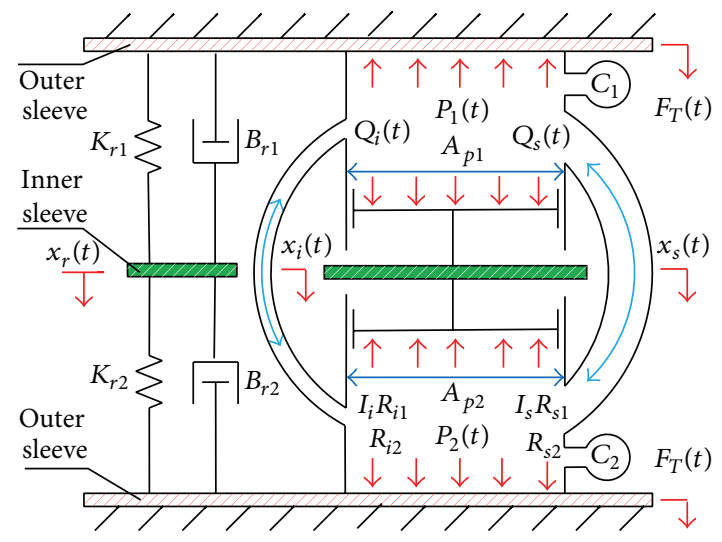

(b)

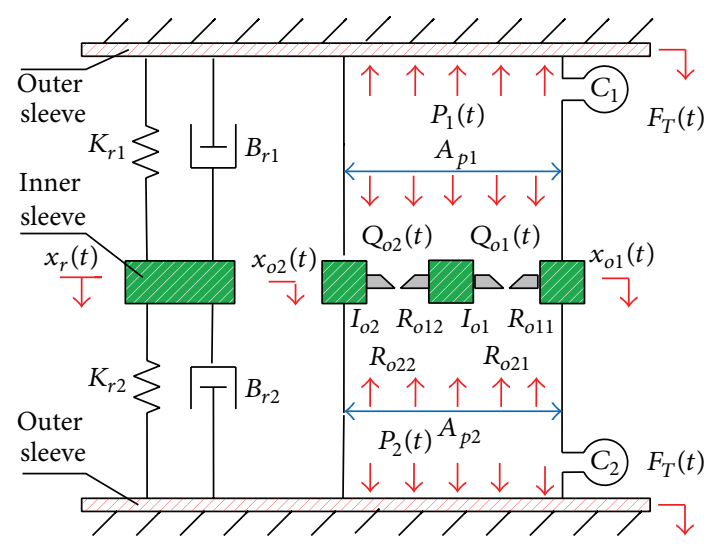

(d)

FIGURE 3: Fluid system model for HDBs with multiple configurations. (a) One inertia track; (b) two inertia tracks; (c) one inertia and an orifice; (d) two orifices.

that of an artificial cylinder piston. $P_{1}(t)$ and $P_{2}(t)$ denote the pressures in the upper and the lower chambers. The inertia tracks and orifices are characterized by the fluid inertial coefficients $I_{i}$ and $I_{o}$ and fluid resistances $R_{i}$ and $R_{o}$, respectively. The static preload and a sinusoidal displacement excitation, $x_{r}(t)=X_{r} \sin \omega t$, are applied to the inner metal sleeve, while the outer metal sleeve is fixed. $F_{T}(t)$ is the dynamic force which is transmitted to the outer metal sleeve by the hydraulic and main rubber paths [4-8].

The static and dynamic tests for the above-mentioned HDB with multiple internal configurations are conducted using elastomer test machine (MTS 831). Detailed descriptions of the test method and data processing technique for obtaining the dynamic properties of an HDB refer to [24]. In addition, it is assumed that the relative displacement between the inner and outer metal sleeves varies in radial direction. Therefore, only radial damping is considered in this study.

2.2. Experimental Results. Figure 4 shows the tests results of static stiffness of the HDBs with different internal configurations, that is, HB1, HB2, HB4, and HB5. It can be seen that the force-displacement curves for these 4 samples are linear and have similar gradients under small amplitude excitations $(0 \sim$ $3 \mathrm{~mm}$ ). However, they tend to differ with each other and be nonlinear for large displacement excitations $(>3 \mathrm{~mm})$. When the displacement excitation is greater than $4 \mathrm{~mm}$, the outer 


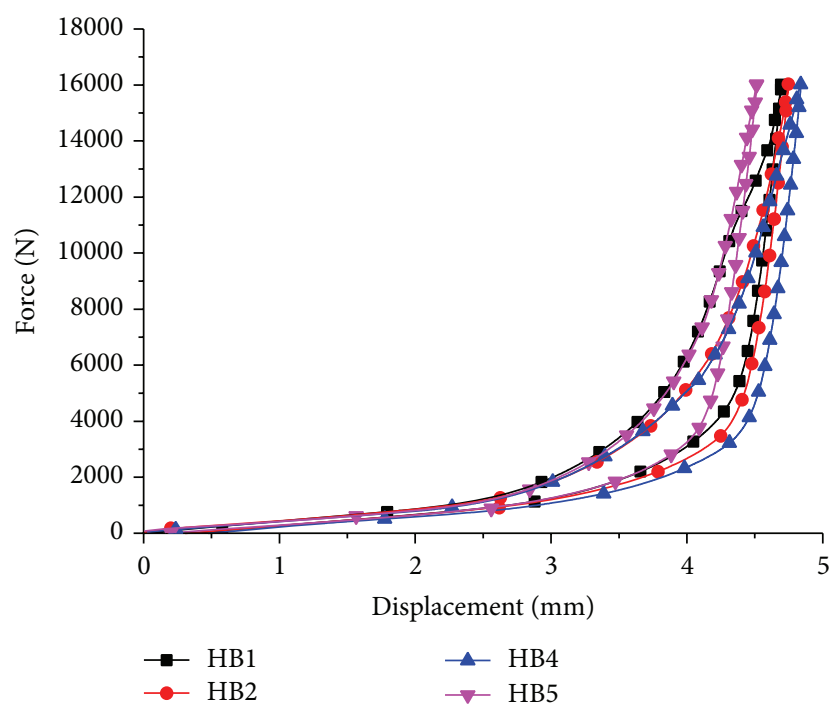

Figure 4: The tests results static characteristics of the HDBs with different internal configurations.

metal sleeve contacts with the stopper member; thus, the force-displacement curves exhibit significant nonlinearity.

In general, the static characteristics of these samples correlated well with each other. This indicates that the vulcanization process conditions for these samples are stable and consistent.

The results of the dynamic tests of the main rubber and the HDBs with multiple internal configurations under sinusoidal excitations are shown in Figure 5.

\subsubsection{The Dynamic Performances of the Main Rubber Spring.} As shown in Figures 5(a) and 5(b), a sinusoidal excitation is applied to the inner sleeve of the main rubber (HB6) with a preload of $387 \mathrm{~N}$. The changes of the dynamic stiffness and loss angle of HB6 with excitation frequency are very small compared to those of HB1, HB2, HB3, HB4, and HB5. Therefore, the dynamic stiffness and the loss angle of the HB6 can be reasonably treated as two constants, for example, taking the average of the test data $\left(K_{r}\right.$ is about $438340 \mathrm{~N} / \mathrm{m}$ and $B_{r}$ is about $102.06 \mathrm{Ns} / \mathrm{m}$ ), in the subsequent analysis.

2.2.2. The Dynamic Performances of HDBs with Multiple Configurations. Figure 5 shows that the dynamic performances of the HDBs with multiple configurations are strongly excitation frequency and amplitude dependent.

As the excitation frequency increases, the dynamic stiffness of both HB3 and HB6 can be considered as constant. The difference between them can be attributed to the incompressibility of the liquid, which causes a stiff. HDB. The dynamic stiffness of HDBs with multiple configurations tends to be a horizontal line under high frequency band, respectively, so they can be considered as amplitude invariant in this scenario.

Under the $0.8 \mathrm{~mm}$ excitation, the frequencies of the peak for the loss angles of HB1, HB2, HB4, and HB5 are 12, 20, 52 , and $88 \mathrm{~Hz}$, respectively. As the frequency increases, the loss angles of HB1, HB2, HB4, and HB5 gradually approach a horizontal line which overlaps the curves of HB3 and HB6. This phenomenon indicates that, in higher frequency range, the response of the fluid in tracks is roughly attenuated and the damping of the HDB is mainly determined by its main rubber. The bandwidths of the loss angle for the HDBs with different internal configurations are different. With the increase of the number of inertia tracks, the HDB can provide damping over a wider frequency band. While increasing the number of orifices, the HDB can provide larger damping in a higher and wider frequency band.

The high frequency dynamic performances of the HDBs with multiple internal configurations can be examined by observing Figures 5(c) and 5(d). As the number of orifices increases, the notch frequency $f_{K_{d} \text { min }}$ shifts to a higher value and the level of the dynamic stiffness $K_{d}$ decreases simultaneously. Moreover, it is shown that the corresponding loss angles are all $90^{\circ}$ which means there is a resonance at $f_{K_{d} \min }$.

It can be found that the loss angle shifts abruptly from about $0^{\circ}$ to $180^{\circ}$. This means the resonance is dominated not only by the fluid flow resistance but also by the fluid inertial effect, suggesting that $\mathrm{HDB}$ with multiple configurations cannot be modelled by a single degree of freedom (SDOF) system under high frequency small amplitude excitation [25, 27]. Since the fluid motion is highly turbulent at higher frequencies, its influence is not modelled here.

2.2.3. The Fixed Points on Dynamic Performances of HDBs with Multiple Configurations. It should be noted that many excitation amplitude-invariant fixed points $M_{i}(i=1 \sim 4)$ and $N_{i}(i=1 \sim 6)$ exist on the dynamic test results curves. With the number of inertia tracks increases, the dynamic stiffness $\left(K_{d M_{i}}\right)$ of the fixed point is decreased. As the number of orifices increases, $K_{d M_{i}}$ decreases rapidly. These fixed points contain important information of an HDB, and a new parameter identification method can be developed.

\section{The Nonlinear LP Model for an HDB with One Inertia Track}

This section presents the modeling of the HDB with one inertial track illustrated in Figure 3(a). The following assumptions are made [29]: (i) the influence of gravity is ignored and the fluid in chamber is incompressible; (ii) the inertia and damping of the fluid in lower and upper chambers are neglected and the fluid pressure in each chamber is uniform; (iii) the relative velocity of fluid is constant along the inertia channel and the properties of inlet and outlet cross sections are uniform; (iv) the dynamic viscosity and density properties of the liquid are constant, and the cross sections along inertia track have the same shape.

With the above assumptions, the continuity equations can be written as

$$
\begin{aligned}
& K_{1}\left(A_{i} x_{i}-A_{p 1} x_{r}\right)=P_{1} \\
& K_{2}\left(A_{p 2} x_{r}-A_{i} x_{i}\right)=P_{2} .
\end{aligned}
$$



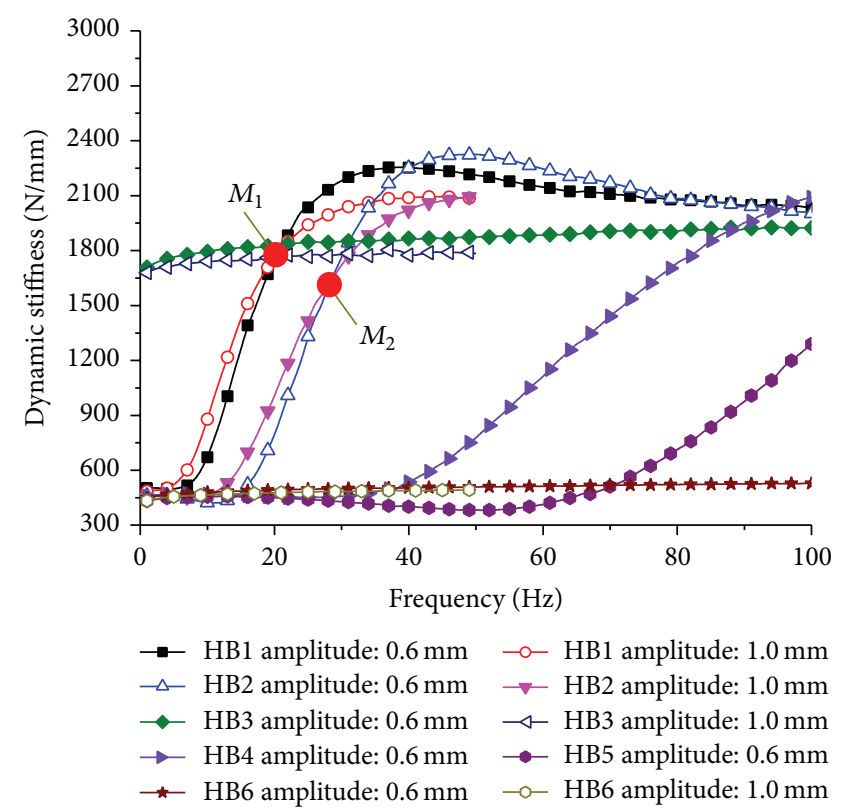

(a)

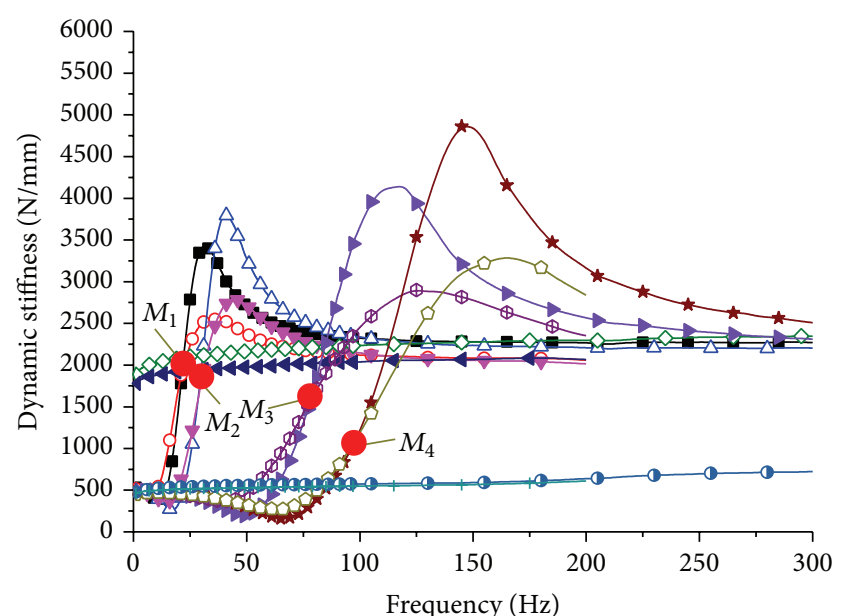

$\rightarrow$ HB1 amplitude $=0.1 \mathrm{~mm} \quad \multimap-\mathrm{HB} 1$ amplitude $=0.3 \mathrm{~mm}$

$\neg$ HB2 amplitude $=0.1 \mathrm{~mm} \rightarrow \mathrm{HB} 2$ amplitude $=0.3 \mathrm{~mm}$

$\checkmark$ HB3 amplitude $=0.1 \mathrm{~mm} \leftarrow$ HB3 amplitude $=0.3 \mathrm{~mm}$

$\rightarrow \quad$ HB4 amplitude $=0.1 \mathrm{~mm} \rightarrow \mathrm{HB} 4$ amplitude $=0.3 \mathrm{~mm}$

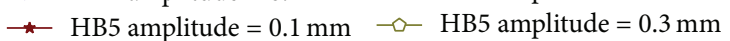

$\multimap$ HB6 amplitude $=0.1 \mathrm{~mm} \rightarrow \mathrm{HB} 6$ amplitude $=0.3 \mathrm{~mm}$

(c)

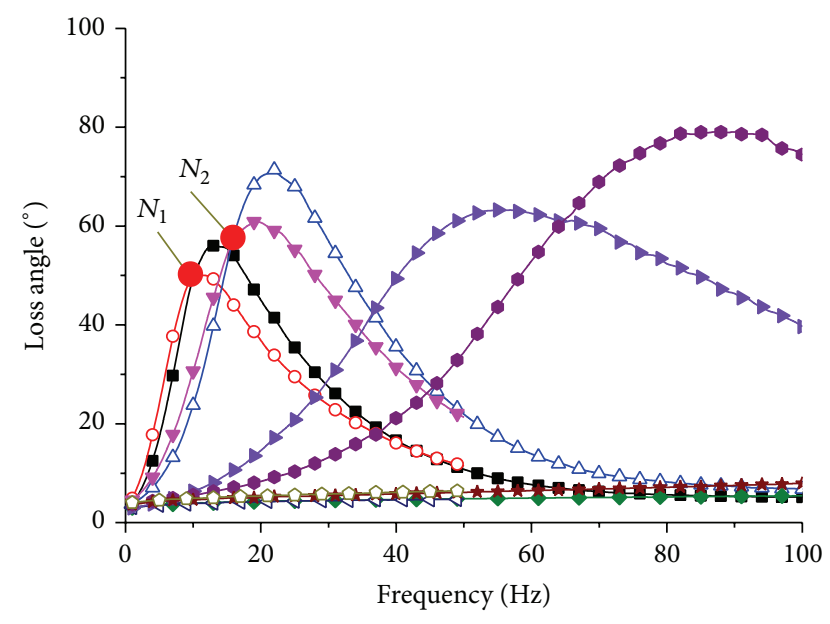

$\rightarrow$ HB1 amplitude: $0.6 \mathrm{~mm} \quad \multimap-\mathrm{HB} 1$ amplitude: $1.0 \mathrm{~mm}$

$\triangle$ HB2 amplitude: $0.6 \mathrm{~mm} \rightarrow \mathrm{HB} 2$ amplitude: $1.0 \mathrm{~mm}$

$\multimap$ HB3 amplitude: $0.6 \mathrm{~mm} \quad \neg$ HB3 amplitude: $1.0 \mathrm{~mm}$

$\rightarrow$ HB4 amplitude: $0.6 \mathrm{~mm} \rightarrow$ HB5 amplitude: $0.6 \mathrm{~mm}$

* HB6 amplitude: $0.6 \mathrm{~mm} \quad \neg-\mathrm{HB} 6$ amplitude: $1.0 \mathrm{~mm}$

(b)

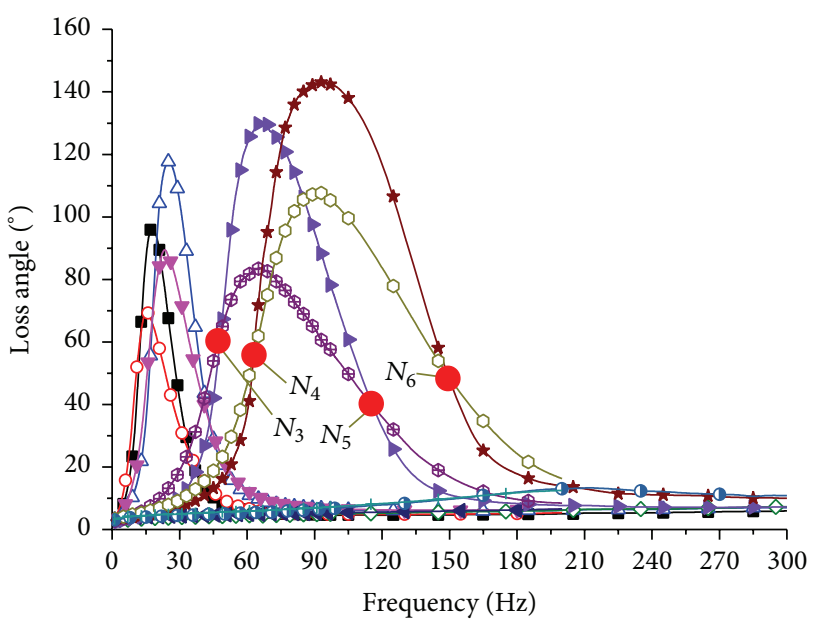

$\rightarrow$ HB1 amplitude $=0.1 \mathrm{~mm} \quad \multimap-\mathrm{HB} 1$ amplitude $=0.3 \mathrm{~mm}$

$\neg-$ HB2 amplitude $=0.1 \mathrm{~mm} \rightarrow$ HB2 amplitude $=0.3 \mathrm{~mm}$

$\checkmark$ HB3 amplitude $=0.1 \mathrm{~mm} \multimap$ HB3 amplitude $=0.3 \mathrm{~mm}$

$\rightarrow$ HB4 amplitude $=0.1 \mathrm{~mm} \rightarrow$ HB4 amplitude $=0.3 \mathrm{~mm}$

\# HB5 amplitude $=0.1 \mathrm{~mm} \quad-\mathrm{O}-\mathrm{HB} 5$ amplitude $=0.3 \mathrm{~mm}$

$\multimap$ HB6 amplitude $=0.1 \mathrm{~mm} \rightarrow \mathrm{HB} 6$ amplitude $=0.3 \mathrm{~mm}$

(d)

FIGURE 5: The dynamic test results of HDBs with different internal configurations. (a) Dynamic stiffness under low frequency band, large amplitude excitations; (b) loss angle under low frequency bands, large amplitude excitations; (c) dynamic stiffness under high frequency band, small amplitude excitations; (d) loss angle under high frequency band, small amplitude excitations.

According to Bernoulli equation for nonstationary flow [30], the momentum equation of the fluid column mass in the inertia track is yielded as follows:

$$
\begin{aligned}
\Delta P & =P_{1}-P_{2}=\rho l_{i} \ddot{x}_{i}+\left(\eta_{l} \frac{l_{i}}{d_{H}}+\xi\right) \frac{\rho}{2}\left|\dot{x}_{i}\right| \dot{x}_{i} \\
\eta_{l} & =\frac{64 \mu \gamma}{\rho\left|\dot{x}_{i}\right| d_{H}}
\end{aligned}
$$

$$
\begin{aligned}
\xi & =\xi_{\text {in }}+\xi_{\text {out }}+\xi_{l} \\
\xi_{\text {in }} & =\frac{1}{2\left(1-A_{\text {out }} / A_{\text {in }}\right)} \\
\xi_{\text {out }} & =\frac{1}{2\left(A_{\text {out }} / A_{\text {in }}-1\right)},
\end{aligned}
$$

where $\eta_{l}$ is the major loss coefficient and the calculation details of $\eta_{l}$ can be found in Wang et al. [29] and $\xi$ is the total local loss factor including contraction loss coefficient of inlet 
$\xi_{\text {in }}$, expansion loss coefficient of outlet $\xi_{\text {out }}$, and the track bend local loss factor $\xi_{l}$ determined by the curvature radius and diameter of fluid track according to empirical formulas [29]. $A_{\text {in }}$ and $A_{\text {out }}$ are the cross-sectional areas of inlet and outlet, respectively.

The dynamic force transmitted to the outer sleeve can be expressed as

$$
\begin{aligned}
F_{T}(t) & =F_{T r}(t)+F_{T h}(t) \\
& =\left(K_{r} x_{r}+B_{r} \dot{x}_{r}\right)+\left(A_{p 2} P_{2}-A_{p 1} P_{1}\right),
\end{aligned}
$$

where $F_{T r}(t)$ is the transmitted force of the rubber path and $F_{T h}(t)$ is the transmitted force of the hydraulic path.

3.1. Analytical Analysis of Dynamic Performance of an HDB with One Inertia Track. Substituting (1) into (2a), (2b), and (2c), the equation of the liquid column flowing in the inertia track can be obtained

$$
\begin{aligned}
\ddot{x}_{i} & +\frac{1}{\rho l_{i}}\left(\eta_{l} \frac{l_{i}}{d_{H}}+\xi\right) \frac{\rho}{2}\left|\dot{x}_{i}\right| \dot{x}_{i}+\frac{1}{\rho l_{i}}\left(K_{2} A_{i}+K_{1} A_{i}\right) x_{i} \\
& =\frac{1}{\rho l_{i}}\left(K_{2} A_{p 2} x_{r}+K_{1} A_{p 1} x_{r}\right) .
\end{aligned}
$$

Then, the resonance frequency of liquid column flowing in the inertia track can be derived as follows:

$$
\omega_{n}=\sqrt{\frac{K_{2} A_{i}+K_{1} A_{i}}{\rho l_{i}}}=2 \pi f_{n} .
$$

Equation (5) indicates that the value of the resonant frequency $f_{n}$ closely relates not only to the bulk stiffness values of two chambers, but also to $\rho, l_{i}, A_{i}$.

Since $\left|\dot{x}_{i}\right| \dot{x}_{i}$ is a quadratic function [31] of the liquid column velocity, the exact solution of (4) cannot be achieved. An alternative is to linearize the nonlinear term as $\left|\dot{x}_{i}\right| \dot{x}_{i} \approx$ $8 X_{i} \omega \dot{x}_{i} / 3 \pi$ utilizing the describing function analysis [31]. Then, (4) can be rewritten as

$$
\ddot{x}_{i}+2 \zeta \omega_{n} \dot{x}_{i}+\omega_{n}^{2} x_{i}=\frac{1}{\rho l_{i}}\left(K_{2} A_{p 2} x_{r}+K_{1} A_{p 1} x_{r}\right),
$$

where the damping ratio of the system is

$$
\zeta=\frac{1}{2 \omega_{n}}\left(\frac{32 \mu \gamma}{\rho d_{H}^{2}}+\frac{4 \xi}{3 \pi l_{i}} \omega X_{i}\right) .
$$

Substituting $x_{r}=X_{r} e^{j \omega t}, x_{i}=X_{i} e^{j\left(\omega t-\varphi_{1}\right)}$, and $F_{T}=$ $\widetilde{F}_{T} e^{j(\omega t-\varphi)}$ into (6), it gives

$$
\begin{aligned}
& \frac{X_{i}}{X_{r}} e^{-j \varphi_{1}}\left[-\omega^{2}+\omega_{n}^{2}+\left(\frac{32 \mu \gamma}{\rho d_{H}^{2}}+\frac{4 \xi}{3 \pi l_{i}} \omega X_{i}\right) j \omega\right] \\
& \quad=\frac{1}{\rho l_{i}}\left(K_{2} A_{p 2}+K_{1} A_{p 1}\right) .
\end{aligned}
$$

Therefore, the transfer function of the fluid flowing in an inertia track can be written as

$$
\begin{aligned}
H_{f} & =\frac{X_{i}}{X_{r}} e^{-j \varphi_{1}} \\
& =\frac{K_{2} A_{p 2}+K_{1} A_{p 1}}{K_{2} A_{i}+K_{1} A_{i}} \frac{1}{1-\lambda^{2}+j\left(G \lambda / \omega_{n}+N X_{i} \lambda^{2}\right)},
\end{aligned}
$$

where

$$
\begin{aligned}
G & =\frac{32 \mu \gamma}{\rho d_{H}^{2}} \\
N & =\frac{4 \xi}{3 \pi l_{i}} \\
\lambda & =\frac{\omega}{\omega_{n}} .
\end{aligned}
$$

Using (1)-(3) and (9), the sinusoidal complex stiffness can be obtained

$$
\begin{aligned}
K_{d}^{*}(j \omega)= & K_{r}+j \omega B_{r}+K_{2} A_{p 2}^{2}+K_{1} A_{p 1}^{2} \\
& -\left(K_{2} A_{p 2} A_{i}+K_{1} A_{p 1} A_{i}\right) H_{f} .
\end{aligned}
$$

Thus, the complex stiffness can be rewritten as

$$
\begin{aligned}
K_{d}^{*}(j \omega)= & \frac{F_{T}(j \omega)}{x_{r}(j \omega)}=\frac{F_{T r}(j \omega)+F_{T f}(j \omega)}{x_{r}(j \omega)} \\
= & K_{r}^{*}+K_{f}^{*}=K^{\prime}+j K^{\prime \prime} \\
K_{r}^{*}= & K_{r}+j \omega B_{r} \\
K_{f}^{*}= & K_{2} A_{p 2}^{2}+K_{1} A_{p 1}^{2} \\
& -\left(K_{2} A_{p 2} A_{i}+K_{1} A_{p 1} A_{i}\right) H_{f},
\end{aligned}
$$

where $K_{r}^{*}$ is the dynamic stiffness of the rubber spring and $K_{f}^{*}$ represents dynamic characteristic induced by the subsystem of chambers and liquid in the inertia track; $K^{\prime}$ represents the storage dynamic stiffness and $K^{\prime \prime}$ denotes the loss dynamic stiffness. According to (12) and (13), the dynamic performances of HDB with one inertia track can be investigated analytically.

3.1.1. When the Ratio of Excitation Frequency and Resonance Frequency of Liquid Column Tends to 0. When the excitation frequency tends to zero, that is, $\lambda \rightarrow 0, H_{f}$ and $K_{f}^{*}$ become

$$
\begin{aligned}
& H_{f(\lambda \rightarrow 0)}=\frac{K_{2} A_{p 2}+K_{1} A_{p 1}}{K_{2} A_{i}+K_{1} A_{i}} \\
& K_{f(\lambda \rightarrow 0)}^{*}=\frac{K_{2} K_{1}\left(A_{p 2}-A_{p 1}\right)^{2}}{K_{2}+K_{1}} .
\end{aligned}
$$

When $A_{p 2} \approx A_{p 1}$, then

$$
\begin{aligned}
& K_{f(\lambda \rightarrow 0)}^{*}=\frac{K_{2} K_{1}\left(A_{p 2}-A_{p 1}\right)^{2}}{K_{2}+K_{1}} \approx 0 \\
& K_{d(\lambda \rightarrow 0)} \approx K_{r(\lambda \rightarrow 0)} .
\end{aligned}
$$

As shown in (14), $H_{f(\lambda \rightarrow 0)}$ is positive, which indicates that the displacement excitation $x_{r}$ and the corresponding response $x_{i}$ are in-phase, and the liquid pumped by the equivalent piston area of the rubber spring is pumped into 
the other chamber in-phase. Thus, there is almost no pressure fluctuation in both chambers. Equation (15) means that the dynamic performance of the HDB is mainly contributed by the rubber spring.

In practice, $A_{p 2} \approx A_{p 1}, K_{f}^{*}$ is not equal to zero, and the value of $K_{d(\lambda \rightarrow 0)}$ is slightly bigger than that of $K_{r(\lambda \rightarrow 0)}$. As the test results shown, when the excitation amplitude is $1.00 \mathrm{~mm}$, $K_{d(f=0)}=485.47 \mathrm{~N} / \mathrm{mm}, K_{r(f=0)}=434.14 \mathrm{~N} / \mathrm{mm}$, and the ratio of $K_{d(f=0)}$ and $K_{r(f=0)}$ is 1.12; that is, they are almost the same. Therefore, $K_{r}$ can be obtained by testing $K_{d}^{*}$ of an HDB with the liquid under a very low frequency excitation.

\subsubsection{When the Ratio of Excitation Frequency and Resonance} Frequency of Liquid Column Tends to 1. Using (9), when the excitation frequency approaches the resonance frequency $\left(f_{n}\right)$ of the inertia track subsystem, that is, $\lambda \rightarrow 1, H_{f}$ and $K_{f}^{*}$ become

$$
\begin{aligned}
& H_{f(\lambda \rightarrow 1)}=\frac{X_{i}}{X_{r}} e^{-j \varphi_{1}} \\
& =-j \frac{K_{2} A_{p 2}+K_{1} A_{p 1}}{\omega_{n} \rho l_{i}} \frac{1}{\left(G+\omega_{n} N X_{i}\right)} \\
& K_{f(\lambda \rightarrow 1)}^{*} \\
& =K_{2} A_{p 2}^{2}+K_{1} A_{p 1}^{2} \\
& \quad+j \frac{\left(K_{2} A_{p 2}+K_{1} A_{p 1}\right)^{2}}{\left(K_{2}+K_{1}\right)} \frac{2 \omega_{n}}{G+\sqrt{G^{2}+4 N X_{r} E}} \\
& e^{-j \varphi_{1}}=\cos \varphi_{1}-j \sin \varphi_{1} \\
& E=\frac{\left(K_{2} A_{p 2}+K_{1} A_{p 1}\right)}{\rho l_{i}} .
\end{aligned}
$$

Substituting (17a) into (11) leads to

$$
\begin{aligned}
& K_{d(\lambda \rightarrow 1)}^{*}=K_{r}^{*}+K_{f}^{*}=K_{r}+K_{2} A_{p 2}^{2}+K_{1} A_{p 1}^{2} \\
& +j\left(\omega B_{r}\right. \\
& \left.+\frac{\left(K_{2} A_{p 2}+K_{1} A_{p 1}\right)^{2}}{\left(K_{2}+K_{1}\right)} \frac{2 \omega_{n}}{G+\sqrt{G^{2}+4 N X_{r} E}}\right) .
\end{aligned}
$$

Using (18), the loss angle of the HDB with one inertia track can be obtained as

$$
\begin{aligned}
& \varphi_{(\lambda \rightarrow 1)} \\
& =\arctan \left(\frac{\omega C_{r}\left(K_{2}+K_{1}\right)+\left(K_{2} A_{p 2}+K_{1} A_{p 1}\right)^{2} \tau_{f}}{\left(K_{2}+K_{1}\right)\left(K_{r}+K_{2} A_{p 2}^{2}+K_{1} A_{p 1}^{2}\right)}\right) \\
& \tau_{f}=\frac{2 \omega_{n}}{\left(G+\sqrt{G^{2}+4 N X_{r} E}\right)} .
\end{aligned}
$$

As shown in (10), (19), many parameters such as $\mu, A_{i}, \xi$, $l_{i}$, and $d_{H}$ can be selected to adjust the loss angle. The smaller the excitation displacement amplitude $X_{r}$, the larger the $\tau_{f}$, and $\varphi_{(\lambda=1)}$ became larger too. This is the reason why HDB has amplitude-variant dynamic performances. This conclusion matches well the experimental results shown in Figure 5(b).

3.1.3. When the Ratio of Excitation Frequency and Resonance Frequency of Liquid Column Tends to $\infty$. Using (9) and (13), when the high excitation frequency is applied on an HDB with one inertia track, that is, $\lambda \rightarrow \infty, H_{f}$ and $K_{f}^{*}$ become

$$
\begin{aligned}
H_{f(\lambda \rightarrow \infty)} & \longrightarrow 0 \\
K_{f(\lambda \rightarrow 1)}^{*} & =K_{2} A_{p 2}^{2}+K_{1} A_{p 1}^{2} .
\end{aligned}
$$

As shown in (20), the displacement response of liquid column is drastically weakened; this effect is sometimes referred to as flow "shut-off" [27] under high excitation frequency. In this situation, the liquid pumped by the piston of the rubber spring is conformed all by two chambers due to their elasticity of bulk, which results in pressure fluctuations.

Substituting (20) into (11), it can be obtained that

$$
K_{d(\lambda \rightarrow \infty)}^{*}=K_{r}+K_{2} A_{p 2}^{2}+K_{1} A_{p 1}^{2}+j \omega B_{r} .
$$

As shown in (21), the storage dynamic stiffness under high excitation frequency is not varied, and $K_{d(\lambda \rightarrow \infty)}$ is constant considering that the influence of the damping of the main rubber is negligible [28], which means that these parameters $K_{r}, K_{1}, K_{2}, A_{p 1}$, and $A_{p 2}$ can be regarded as constants. This analysis result agrees well with the test results shown in Figure 5(a).

\subsection{Parameter Identification Method Based on the Fixed Points.} Comparing (18) with (21), then

$$
K_{(\lambda \rightarrow 1)}^{\prime}=K_{(\lambda \rightarrow \infty)}^{\prime} \approx K_{r}+K_{2} A_{p 2}^{2}+K_{1} A_{p 1}^{2} .
$$

It can be seen that the storage dynamic stiffness tends to be a constant in higher frequency range and the values of $K_{(\lambda \rightarrow 1)}^{\prime}$ and $K_{(\lambda \rightarrow \infty)}^{\prime}$ are identical. This indicates that the storage dynamic stiffness curves for different amplitude excitations will intersect at $f_{n}$ and have the value of $K_{r}+$ $K_{2} A_{p 2}^{2}+K_{1} A_{p 1}^{2}$.

As shown in Figure 6(a), all the tested curves of storage dynamic stiffness of HB1 pass through the fixed point $M_{5}$, and all the curves of storage dynamic stiffness of HB3 pass through the fixed point $M_{5}$ approximately. All the curves almost approach a horizontal line in higher frequency range. Thus, the analytical conclusion is verified by test results, which implies the validity of the nonlinear LP model.

Set the excitation frequency at $M_{5}$ as $f_{m 5}$ and the corresponding storage dynamic stiffness as $K_{(\lambda=1)}^{\prime}$. Based on the above analytical analysis, the frequency $f_{m 5}$ of the fixed point $\left(M_{5}\right)$ and $f_{n}$ of the liquid column have the following relationship:

$$
f_{m 5} \approx f_{n}=\frac{1}{2 \pi} \sqrt{\frac{K_{2} A_{i}+K_{1} A_{i}}{\rho l_{i}}} .
$$




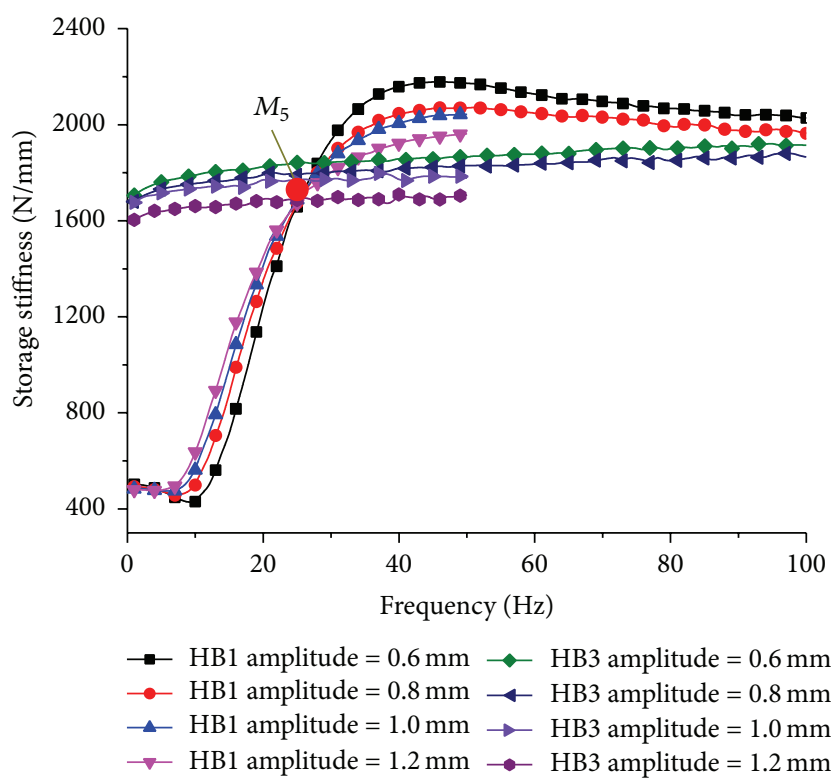

(a)

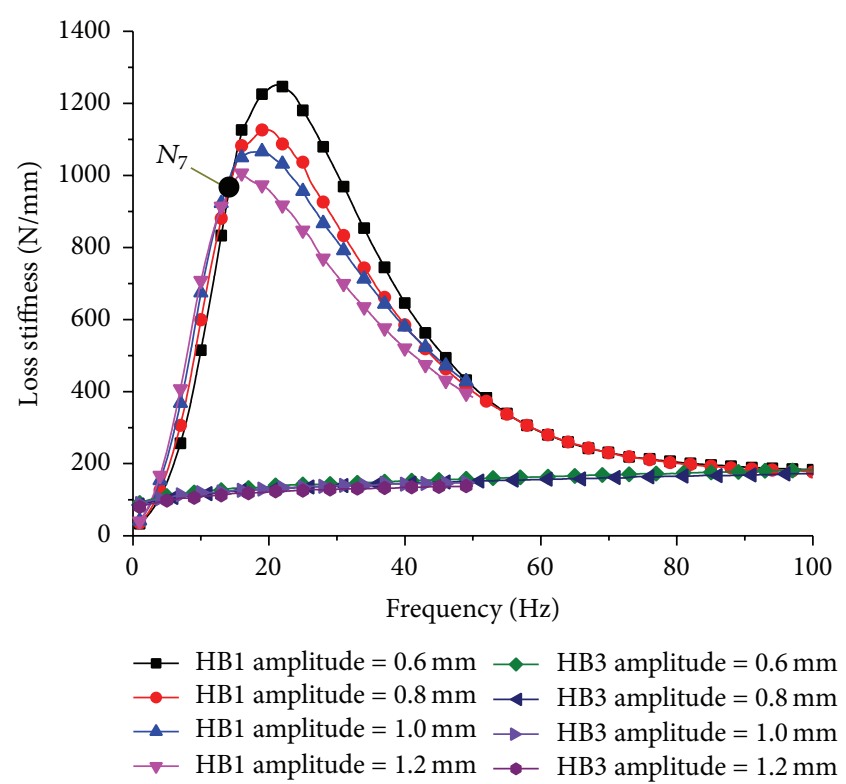

(b)

FIGURE 6: The test results of storage and loss dynamic stiffness of the hydraulic bushing. (a) Storage stiffness. (b) Loss stiffness.

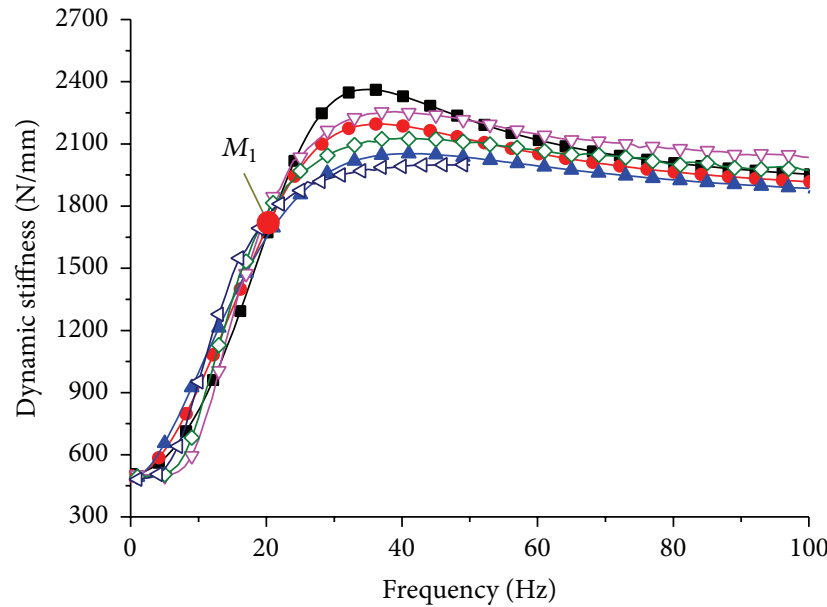

$\rightarrow$ Calculated amplitude $=0.6 \mathrm{~mm} \rightarrow-$ Tested amplitude $=0.6 \mathrm{~mm}$ $\multimap$ Calculated amplitude $=0.8 \mathrm{~mm} \leadsto$ Tested amplitude $=0.8 \mathrm{~mm}$ $\multimap$ Calculated amplitude $=1.2 \mathrm{~mm} \smile-$ Tested amplitude $=1.2 \mathrm{~mm}$

(a)

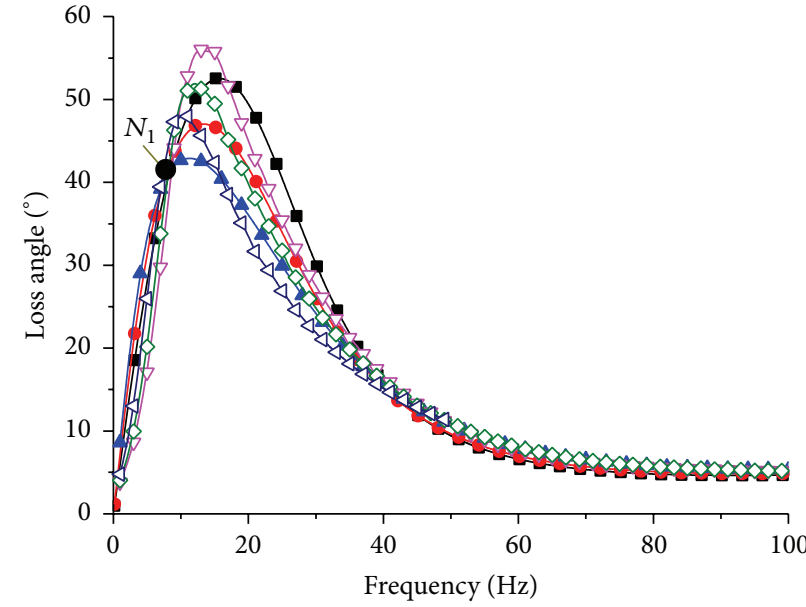

- Calculated amplitude $=0.6 \mathrm{~mm} \rightarrow-$ Tested amplitude $=0.6 \mathrm{~mm}$ $\multimap$ Calculated amplitude $=0.8 \mathrm{~mm} \leadsto$ Tested amplitude $=0.8 \mathrm{~mm}$ $\multimap$ Calculated amplitude $=1.2 \mathrm{~mm} \backsim$ Tested amplitude $=1.2 \mathrm{~mm}$

(b)

Figure 7: Comparisons between experimental and predicted results of the dynamic characteristics of HB1. (a) Dynamic stiffness. (b) Loss angle.

Since the curves near the fixed point $M_{5}$ are almost vertical to the frequency axis as shown in Figure 6(a), small experiment error of the storage dynamic stiffness will not cause a notable change in $f_{m 5}$. Therefore, the experimental data of $f_{m 5}$ can be employed to identify $f_{n}$. Supposing $K_{1}$ and $K_{2}$ are proportional, the values of $K_{1}$ and $K_{2}$ can then be obtained according to (23).

Besides, the curves of storage dynamic stiffness are almost perpendicular to the longitudinal axis when $\lambda \rightarrow \infty$, so a higher variation of excitation frequency will only lead to a nearly smaller fluctuation of storage dynamic stiffness in high frequency range. On the contrary, the slopes of these curves around the resonance frequency (when $\lambda=1$ ) are almost vertical to the frequency axis; thus, a smaller fluctuation of excitation frequency will result in a significant variation of storage dynamic stiffness. So, the parameter identification results for $A_{p 1}$ and $A_{p 2}$ based on $K_{(\lambda \rightarrow \infty)}^{\prime}$ are more precise and credible.

According to the above method and Table 1, these parameters identifications are conducted and the results are summarized as follows: $K_{r}=4.86 \times 10^{5}(\mathrm{~N} / \mathrm{m}), K_{2}=2.31 \times$ $10^{11}\left(\mathrm{~N} / \mathrm{m}^{5}\right), K_{1}=2.08 \times 10^{11}\left(\mathrm{~N} / \mathrm{m}^{5}\right), A_{p 1}=1.91 \times$ $10^{-3}\left(\mathrm{~m}^{2}\right)$, and $A_{p 2}=1.72 \times 10^{-3}\left(\mathrm{~m}^{2}\right)$. Then, the dynamic performances of $\mathrm{HB1}$ are calculated and compared with the experiment data, as shown in Figure 7. It is shown that the calculation results match well the experiment data, which 


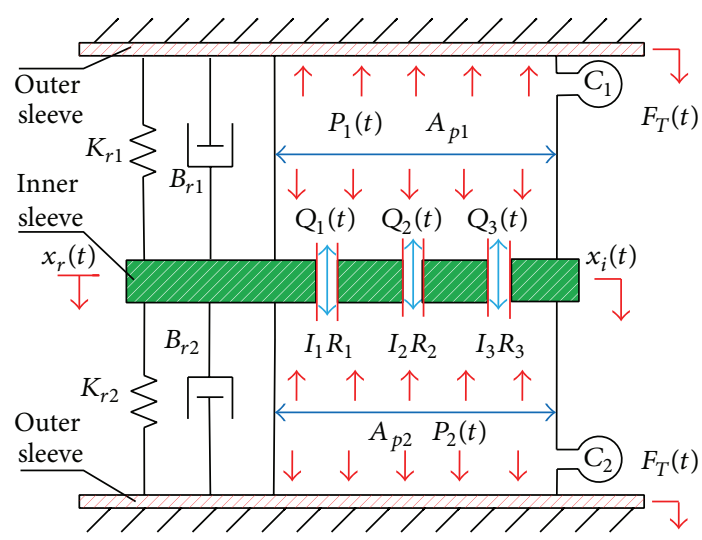

FIgURE 8: Fluid system model for an HDB with three nonidentical inertia tracks.

further validates the analytical analysis presented in this study.

\section{The LP Models for HDB with Multiple Inertia Tracks and Orifices}

As shown in Figure 5, the dynamic performances of HB2, $\mathrm{HB} 4$, and HB5 are very similar to that of HB1. This suggests that the LP model of HDB with one inertia track is also applicable for those with multiple inertial tracks. Thus, it will be adopted in the following analyses.

4.1. The LP Model for an HDB with Nonidentical Inertia Tracks. An LP model of HDB with three nonidentical inertial tracks is illustrated in Figure 8. $A_{i}, l_{i}(i=1,2,3)$ denote the lengths and the cross-sectional areas of the inertia tracks and $i$ represents the inertial track number; $\xi_{i 1}$ and $\xi_{i 2}(i=1,2,3)$ are the linear and nonlinear damping coefficients of the fluid motion in the inertia tracks, respectively.

The inertia $I_{i}$ and the linear and nonlinear resistances $R_{i 1}$ and $R_{i 2}(i=1,2,3)$ of the fluid in the inertia track can be obtained by

$$
\begin{aligned}
I_{i} & =\frac{\rho A_{i}}{l_{i}} \\
R_{i 1} & =\frac{\xi_{i 1}}{A_{i}^{2}} \\
R_{i 2} & =\frac{\xi_{i 2}}{A_{i}^{5}} .
\end{aligned}
$$

The fluid volume flow rate in three inertia tracks can be denoted as $Q_{i}(i=1,2,3)$, respectively. The state equation of the LP model can be written as follows with the state vector defined as $\mathbf{X}^{T}=\left(x_{1}, x_{2}, x_{3}, x_{4}, x_{5}\right)=\left(P_{1}, P_{2}, Q_{1}, Q_{2}, Q_{3}\right)$ :

$$
\dot{\mathbf{X}}=\mathbf{A X}+\mathbf{B},
$$

where

$$
\begin{aligned}
& \mathbf{A}=\left[\begin{array}{ccccc}
0 & 0 & -K_{1} & -K_{1} & -K_{1} \\
0 & 0 & K_{2} & K_{2} & K_{2} \\
\frac{1}{I_{1}} & \frac{-1}{I_{1}} & \frac{-\left(R_{11}+R_{12}\left|x_{3}\right|\right)}{I_{1}} & 0 & 0 \\
\frac{1}{I_{2}} & \frac{-1}{I_{2}} & 0 & \frac{-\left(R_{21}+R_{22}\left|x_{4}\right|\right)}{I_{2}} & 0 \\
\frac{1}{I_{3}} & \frac{-1}{I_{3}} & 0 & 0 & \frac{-\left(R_{31}+R_{32}\left|x_{5}\right|\right)}{I_{2}}
\end{array}\right] \\
& \mathbf{B}=\left[\begin{array}{c}
-K_{1} A_{p 1} \dot{x} \\
K_{2} A_{p 2} \dot{x} \\
0 \\
0 \\
0
\end{array}\right] \text {. }
\end{aligned}
$$

In general, the nonlinear resistances $R_{i 2}(i=1,2,3)$ are negligible; that is, $R_{i 2}=0(i=1,2,3)$. Since $l_{i}(i=1$, $2,3)$ is finite, there will be some end effects due to contraction loss of inlet and expansion loss of outlet, and the tracks are curvilinear. These additional effects (including turbulence) are expected to add more damping to the HDB.
To compensate these effects, an empirical coefficient $\gamma$ is introduced to better approximate the actual system and to enhance the capillary tube resistance $R_{i 1}(i=1,2,3)$ as follows [32]:

$$
R_{i 1}=\gamma \frac{128 \mu l_{i}}{\pi d_{H}^{4}},
$$




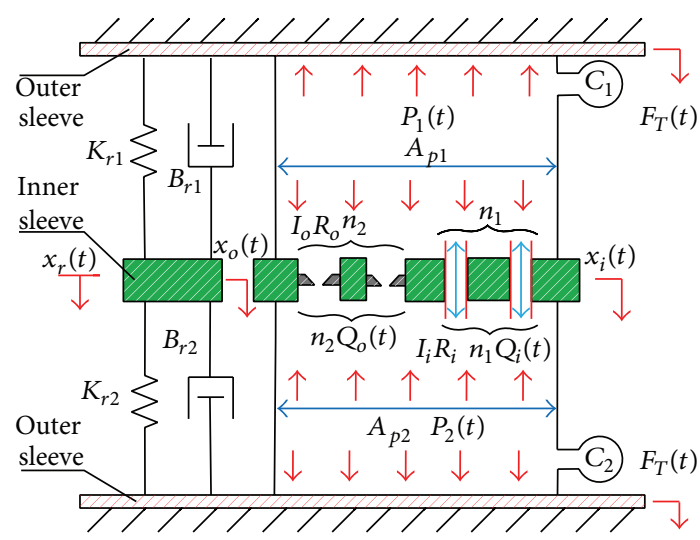

FIGURE 9: Fluid system model of hydraulic bushing with multiple identical inertia tracks and identical orifices.

where $\mu$ is the dynamic viscosity of the HDB fluid and $\mathbf{A}$ then becomes a constant matrix $\mathbf{A}_{L}$ as

$$
\mathbf{A}_{L}=\left[\begin{array}{ccccc}
0 & 0 & -K_{1} & -K_{1} & -K_{1} \\
0 & 0 & K_{2} & K_{2} & K_{2} \\
\frac{1}{I_{1}} & \frac{-1}{I_{1}} & \frac{-R_{11}}{I_{1}} & 0 & 0 \\
\frac{1}{I_{2}} & \frac{-1}{I_{2}} & 0 & \frac{-R_{21}}{I_{2}} & 0 \\
\frac{1}{I_{3}} & \frac{-1}{I_{3}} & 0 & 0 & \frac{-R_{31}}{I_{2}}
\end{array}\right] .
$$

Substituting $\mathbf{Q}^{T}=\left(Q_{1}, Q_{2}, Q_{3}\right)$ and $R_{i 2}=0(i=1,2,3)$ into (25), the motion equation of the lumped fluid mass can be written as

$$
\mathbf{M Q}+\mathbf{C} \dot{\mathbf{Q}}+\mathbf{K Q}=\mathbf{F}(t),
$$

where $\mathbf{M}, \mathbf{C}, \mathbf{K}$, and $\mathbf{F}(t)$ are mass matrix, damping matrix, stiffness matrix, and force vector, respectively, and can be expressed as

$$
\begin{aligned}
\mathbf{M} & =\left[\begin{array}{lll}
I_{1} & & \\
& I_{2} & \\
& & I_{3}
\end{array}\right] \\
\mathbf{C} & =\left[\begin{array}{lll}
R_{11} & & \\
& R_{21} & \\
& & R_{31}
\end{array}\right] \\
\mathbf{K} & =\left[\begin{array}{lll}
K_{1}+K_{2} & K_{1}+K_{2} & K_{1}+K_{2} \\
K_{1}+K_{2} & K_{1}+K_{2} & K_{1}+K_{2} \\
K_{1}+K_{2} & K_{1}+K_{2} & K_{1}+K_{2}
\end{array}\right] \\
\mathbf{F}(t) & =\left[\begin{array}{lll}
-K_{1} A_{p 1} & -K_{2} A_{p 2} & 0 \\
-K_{1} A_{p 1} & -K_{2} A_{p 2} & 0 \\
-K_{1} A_{p 1} & -K_{2} A_{p 2} & 0
\end{array}\right] .
\end{aligned}
$$

The characteristic equation of (29) is

$$
\left|\mathbf{K}-\omega_{n}^{2} \mathbf{M}\right|=0 .
$$

According to (31), the resonance frequency $f_{n}$ can be obtained as

$$
f_{n}=\frac{\omega_{n}}{2 \pi}=\sqrt{\frac{\left(K_{1}+K_{2}\right)}{\rho}\left(\frac{A_{1}}{l_{1}}+\frac{A_{2}}{l_{2}}+\frac{A_{3}}{l_{3}}\right)} .
$$

This indicates that there is only one peak frequency for the loss angle, and the system has only one nonzero resonant frequency.

4.2. The LP Model for an HDB with Multiple Identical Inertia Tracks and Identical Orifices. An LP model for an HDB with two groups of identical inertia tracks and identical orifices is shown in Figure 9. The inertia track group consists of $n_{1}$ identical inertia tracks with the length of $l_{i}$ and crosssectional area of $A_{i}$ for each inertia track. Each inertia track is characterized by the fluid inertances $I_{i}$, linear fluid resistances $R_{i 1}$, and nonlinear resistances $R_{i 2}\left(i=1,2, \ldots, n_{1}\right)$ of the lumped fluid mass. $I_{i}$ can be thought of as the resistance to change in flow rate $\dot{Q}_{i}$ to the pressure gradient $\Delta P$ applied on the fluid element. $R_{i}$ of these elements is fundamentally the energy dissipation of fluid flow $Q_{i}$ over the pressure gradient $\Delta P$ of the element.

Based on the steady flow assumption, the orifice-type models can also be used to describe the damping of orificetype tracks for $R_{o}$ concentrated over a short distance. Because the orifice-type track has an aspect ratio of $2 l_{o} / d_{H} \approx 1$, it can be taken as a short-tube orifice for dynamic performance prediction of HDB. The discharge coefficient $c_{o}$ of such a short-tube orifice has the following empirical formulation [32] based on the Reynolds number (Re):

$$
c_{o}= \begin{cases}\left(1.5+13.74 \frac{l_{o}}{d_{H} \operatorname{Re}}\right)^{-1 / 2} & \frac{d_{H} \mathrm{Re}}{l_{o}}>50 \\ \left(2.28+64 \frac{l_{o}}{d_{H} \operatorname{Re}}\right)^{-1 / 2} & \frac{d_{H} \operatorname{Re}}{l_{o}}<50 .\end{cases}
$$

Using the square-root relation $[32,33]$ and taking into account the impact of the fluid jet, the fluid inertances $I_{o}$ and the linearized resistance $R_{o 1}\left(o=1,2, \ldots, n_{2}\right)$ of an orifice can be expressed as

$$
\begin{aligned}
I_{o} & =\beta \frac{\rho l_{o}}{A_{o}} \\
R_{o 1} & =\varepsilon \frac{\rho Q_{o}}{c_{o}^{2} A_{o}^{2}},
\end{aligned}
$$

where $\beta$ and $\varepsilon$ are empirical coefficients for adjusting HDB's inertances and damping in nonideal cases. $Q_{o}$ denotes the flow through the orifice [32].

Assuming the state variable of the LP model is $\mathbf{X}$ and is defined as $\mathbf{X}^{T}=\left(x_{1}, x_{2}, x_{3}, x_{4}\right)=\left(P_{1}, P_{2}, Q_{i}, Q_{o}\right)$, the state equation then can be obtained as

$$
\dot{\mathbf{X}}=\mathbf{A X}+\mathbf{B},
$$


TABLE 3: Comparison of the liquid column resonance frequency.

\begin{tabular}{lcccc}
\hline Different HDB & HB1 $\left(n_{1}=1, n_{2}=0\right)$ & HB2 $\left(n_{1}=2, n_{2}=0\right)$ & HB4 $\left(n_{1}=1, n_{2}=1\right)$ & HB5 $\left(n_{1}=0, n_{2}=2\right)$ \\
\hline Experiment & $12 \mathrm{~Hz}$ & $20 \mathrm{~Hz}$ & $52 \mathrm{~Hz}$ & $82 \mathrm{~Hz}$ \\
Simulation & $13.5 \mathrm{~Hz}$ & $23.5 \mathrm{~Hz}$ & $58.5 \mathrm{~Hz}$ & $88 \mathrm{~Hz}$ \\
\hline
\end{tabular}

where

A

$$
\mathbf{B}^{T}=\left(-K_{1} A_{p 1} \dot{x}_{r}, K_{2} A_{p 2} \dot{x}_{r}, 0,0\right) .
$$

The dynamic force transmitted to the outer sleeve by the rubber and hydraulic paths is

$$
F_{T}(t)=K_{r} x_{r}(t)+B_{r} \dot{x}_{r}(t)+\left(A_{p 2} P_{2}-A_{p 1} P_{1}\right) .
$$

Accordingly, the transfer function can be derived from (34), (35), and (37) in the Laplace domain as follows:

$$
\begin{aligned}
K_{d}^{*}(s)= & \frac{L[F(t)]}{L\left[x_{r}(t)\right]} \\
= & K_{r}+B_{r} s+A_{p 2}^{2} K_{2}+A_{p 1}^{2} K \\
& -\left[\frac{M^{2} Z_{s}^{2} s^{2}+M^{2}\left[\left(Z_{i} s+n_{1} K\right)\left(Z_{s} s+n_{2} K\right)-n_{2} K^{2}\right]+M^{2} n_{2} K Z_{s} s-M^{2} n_{1} K Z_{s} s}{\left(Z_{s} s+n_{2} K\right)^{2}\left(Z_{i} s+n_{1} K\right)-n_{2}\left(Z_{s} s+n_{2} K\right) K^{2}}\right],
\end{aligned}
$$

where

$$
\begin{aligned}
M & =A_{p 1} K_{1}+A_{p 2} K_{2} \\
Z_{i} & =I_{i} s+R_{i 1} \\
Z_{o} & =I_{o} s+R_{o 1} \\
K & =K_{1}+K_{2} .
\end{aligned}
$$

Using the same technique presented in Section 4.1, the peak frequency of the loss angle $f_{n}$ can be explicitly expressed as

$$
f_{n}=\frac{1}{2 \pi} \sqrt{\frac{\left(K_{1}+K_{2}\right)}{\rho}\left(\frac{n_{1} A_{i}}{l_{i}}+\frac{n_{2} A_{o}}{l_{o}}\right)} \text {. }
$$

\section{Model Verification and Discussion}

5.1. Model Verification. As shown in Figure 9, when $n_{2}=0$ and $n_{1}=1$, the LP model is the same as the fluid system model of an HDB with one inertia track shown in Figure 3(a); when $n_{2}=0$ and $n_{1}=2$, the LP model depicts the fluid system model of an HDB with two inertia tracks shown in Figure 3(b); when $n_{2}=1$ and $n_{1}=1$, the LP model represents the fluid system model of an HDB with one inertia track and one orifice element shown in Figure 3(c); when $n_{2}=2$ and $n_{1}=0$, the LP model denotes the fluid system model of an HDB with two orifices shown in Figure 3(d).
The comparisons between the tested and calculated dynamic performances of HDBs with multiple identical inertia tracks and identical orifices under excitation amplitude of $0.8 \mathrm{~mm}$ and lower frequency band $(1 \sim 100 \mathrm{~Hz})$ are presented in Figure 10. In general, the calculated results agree with the experiment data reasonably. Obvious errors for $\mathrm{HB} 4$ and HB5 can be seen around frequencies of $35 \mathrm{~Hz}$ and $55 \mathrm{~Hz}$, respectively. This mainly attributes to the assumption that the fluid-jet length of the short-tube orifice can be regarded as the length of inertia track, which underestimates the impact of liquid turbulence in the orifice. However, the calculated dynamic performances of HB4 and HB5 can predict the dynamic performances of the HDBs in the whole frequency range. Therefore, the proposed model for an HDB with multiple identical inertia tracks and identical orifices is validated.

Based on the geometric and material parameters of the HDB listed in Table 1 , the peak frequency of the loss angle $f_{n}$ of the HDB with multiple identical inertia tracks and identical orifices can be calculated according to (40). Table 3 shows the calculated results and the corresponding experiment data. It can be seen that they match well each other. Thus, the calculation method is validated.

5.2. Influences of Key Parameters. Based on a lot of parametric analyses of the HDB with one inertia track, the same dynamic performances as that of the HDB with two identical inertia tracks can only be achieved by adjusting the length of the fluid track. 


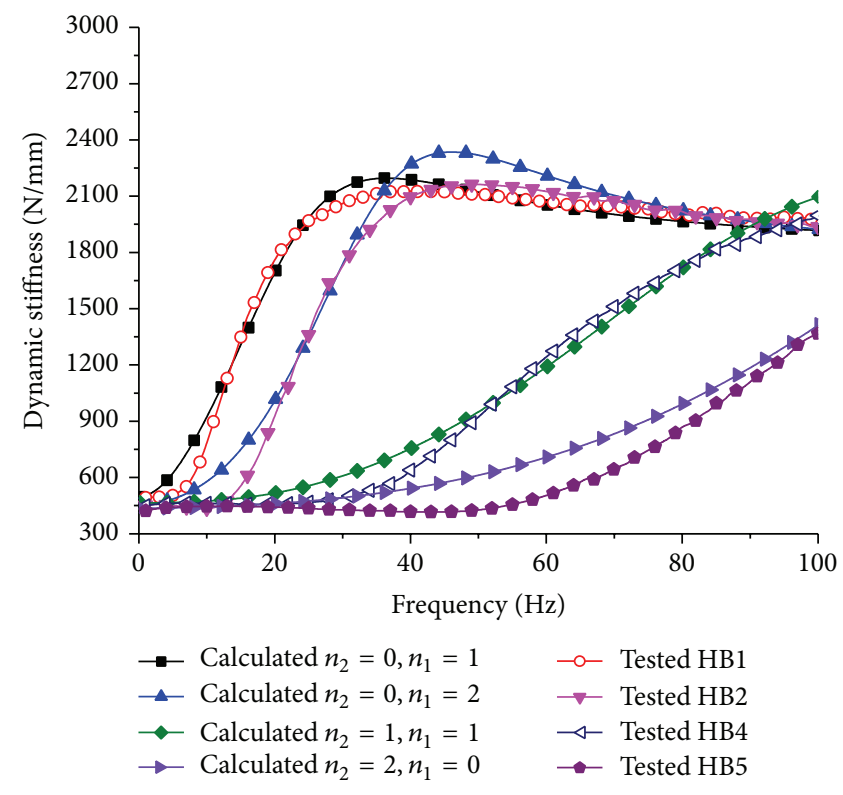

(a)

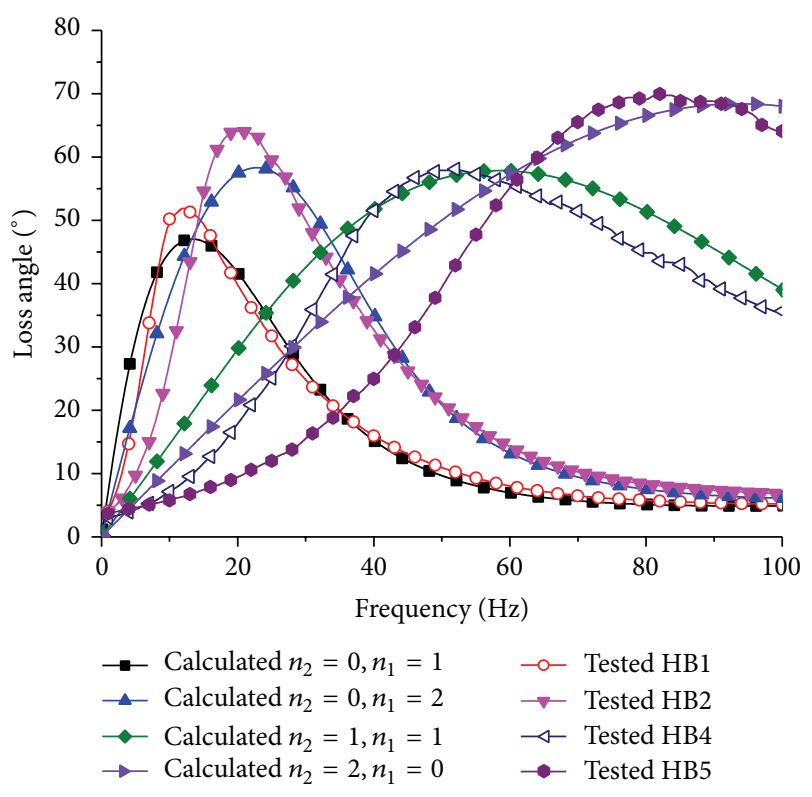

(b)

FIgURE 10: Dynamic performances of HDB (excitation amplitude: $0.8 \mathrm{~mm}$ ). (a) Dynamic stiffness. (b) Loss angle.

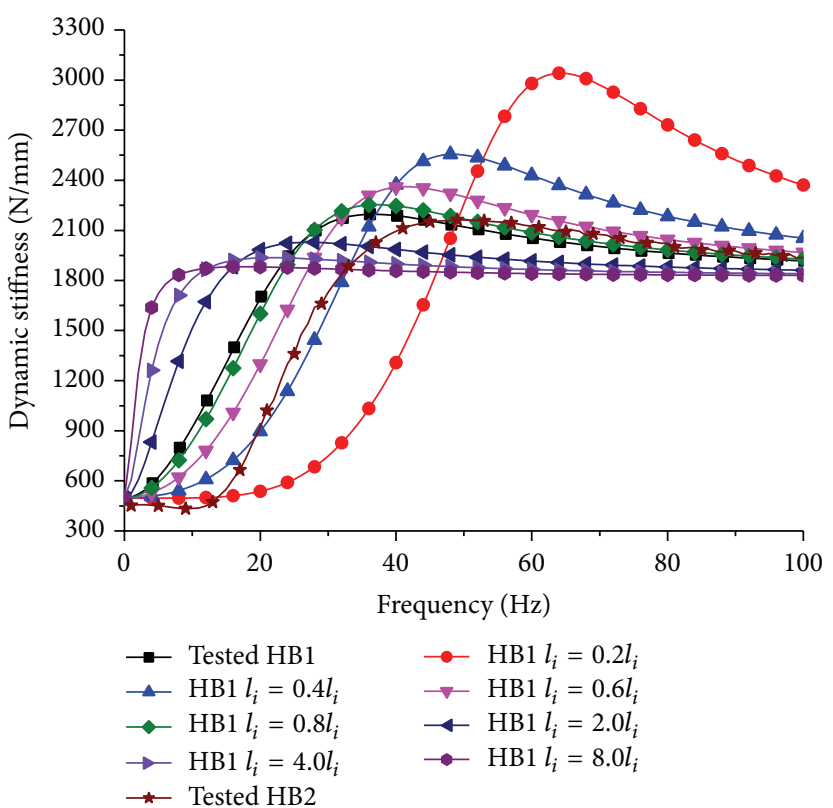

(a)

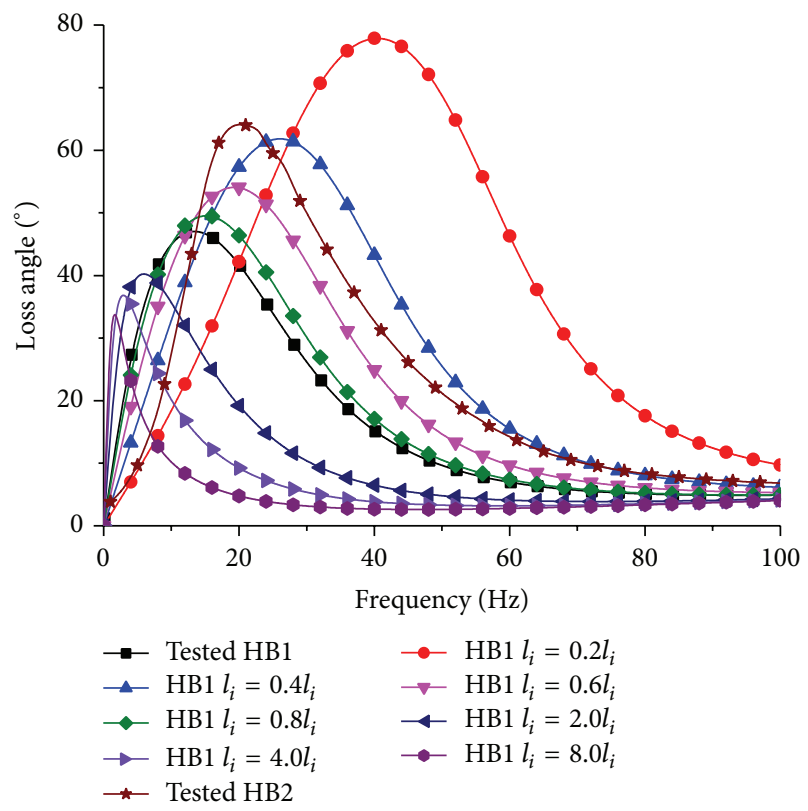

(b)

FIgURE 11: Calculated dynamic performances of HDB (excitation amplitude: $0.8 \mathrm{~mm}$ ). (a) Dynamic stiffness. (b) Loss angle.

As shown in Figure 11, when $0.4 l_{i}<l_{i}<0.6 l_{i}$, the curves of loss angle for HB1 approach that of HB2, and when $0.2 l_{i}<l_{i}<0.6 l_{i}$, the curves of dynamic stiffness for HB1 show consistency with that of HB2. This indicates that the same dynamic performances of an HDB with two identical inertia tracks cannot be achieved simultaneously by only changing the track length of an HDB with one inertia track.

As well known, the HDBs have irregular geometry and are constructed with alternate materials from elastomers to metals. It will be a very challenging work to reach an ideal dynamic performance by changing those parameters, considering the huge numbers of experiments. Varying the number of inertia tracks can be the best choice.

In general, an $\mathrm{HDB}$ with $n_{1} \geq 2$ is more versatile for tuning since there are now many $l_{i}$ and $A_{i}$ to change, but as the number of inertia tracks increases, the maximum dynamic stiffness, the maximum loss angle, and the peak frequency of the loss angle also increase as shown in Figure 12, 


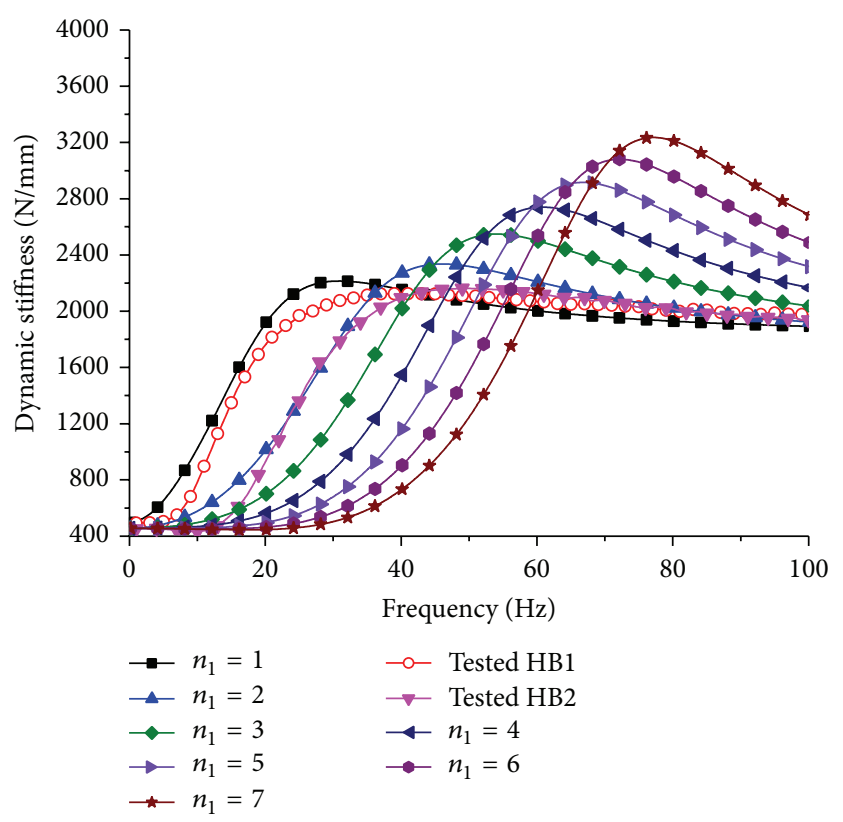

(a)

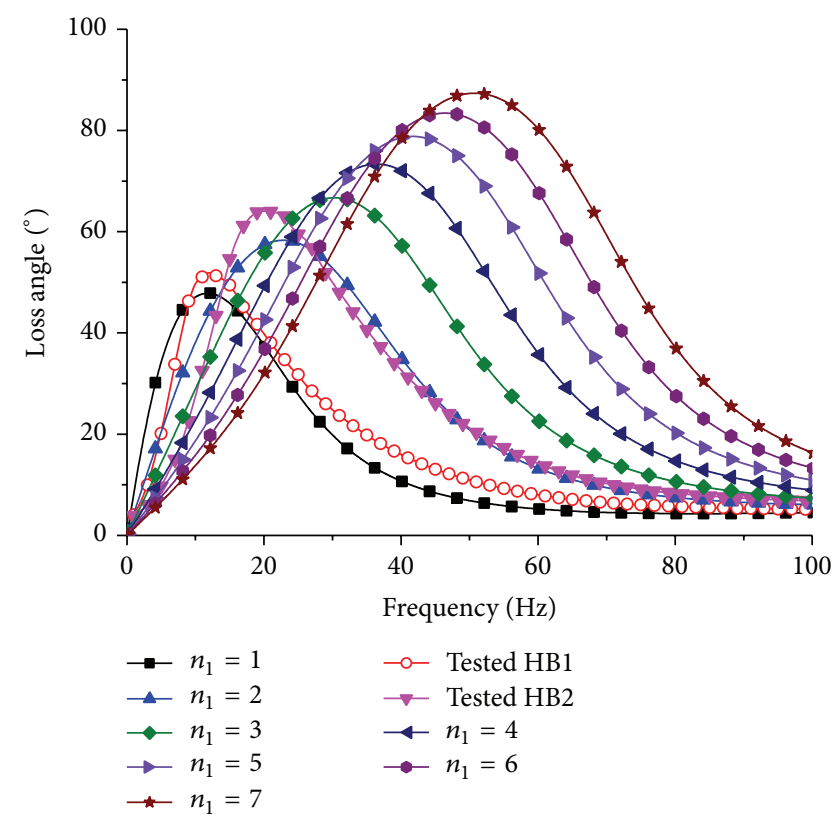

(b)

FIGURE 12: Calculated dynamic performances of HDB with inertia tracks increasing. (a) Dynamic stiffness. (b) Loss angle.

which is good for motion control of, say, the automotive suspension bounce mode.

5.3. Prediction of Dynamic Performances of the HDBs. The influences of the number of inertia tracks on the dynamic performances of an $\mathrm{HDB}$ can be analytically investigated with the LP model proposed in Section 4.2. Assume that an HDB consists of $n_{1}\left(n_{2}=0,1 \leq n_{1} \leq 7\right)$ identical inertia tracks. The influences of the number of inertia tracks on the dynamic performances of the HDB are shown in Figure 12. It is expected that an increase in the number of inertia tracks will introduce more fluid oscillating force contributing to $F_{T}(t)$.

Comparing the results for the inertia track cases of $n_{1}=2$ to $n_{1}=7$ shown in Figure 12, it shows that the maximum dynamic stiffness increases from approximately $2200 \mathrm{~N} / \mathrm{mm}$ to $3200 \mathrm{~N} / \mathrm{mm}$. Additionally, the maximum loss angle increases from approximately $50^{\circ}$ to $87^{\circ}$ and the peak frequency of the loss angle increases from approximately $13.5 \mathrm{~Hz}$ to $52 \mathrm{~Hz}$. Thus, the sensitivity of the HDB's performance increases near the maximum loss angle as well, as the increase of the number of inertia tracks. Further, the number of inertia tracks can be used to tune the device over a wider range of frequencies via increasing the peak frequency of the loss angle.

The influences of the track type on the performances of an HDB can be numerically analyzed with the proposed LP mode in Section 4.2 too. Assume that an HDB consists of $n_{1}$ identical inertia tracks and $n_{2}$ identical orifice tracks. The dynamic performances of these HDBs are shown in Figure 13. When comparing the results for track cases of $n_{1}=1\left(n_{2}=2\right)$ and $n_{1}=2\left(n_{2}=2\right)$ to that for the track case of $n_{1}=3$ $\left(n_{2}=2\right)$, it can be seen that the influences of the number of inertia tracks are not significant since the existence of orifices.
Comparing the results for the inertia track cases of $n_{1}=2$ to $n_{1}=7$ shown in Figure 12, it is shown that the maximum dynamic stiffness increases from approximately $2200 \mathrm{~N} / \mathrm{mm}$ to $3200 \mathrm{~N} / \mathrm{mm}$. The maximum loss angle increases from approximately $50^{\circ}$ to $87^{\circ}$ and the peak frequency of the loss angle increases from approximately $13.5 \mathrm{~Hz}$ to $52 \mathrm{~Hz}$. The HDB's performances near the maximum loss angle become more sensitive as the number of inertia tracks increases. Thus, increasing the number of inertia tracks can improve the device performance over a wider frequency range.

Observing the results for the track case of $n_{1}=0\left(n_{2}=4\right)$ and $n_{1}=0\left(n_{2}=6\right)$ shown in Figure 13, it can be seen that the maximum dynamic stiffness decreases from approximately $2300 \mathrm{~N} / \mathrm{mm}$ to $2220 \mathrm{~N} / \mathrm{mm}$, but the dynamic stiffness increases from approximately $1050 \mathrm{~N} / \mathrm{mm}$ to $1250 \mathrm{~N} / \mathrm{mm}$ at a very low frequency, for example, $1 \mathrm{~Hz}$. In addition, the maximum loss angle decreases from approximately $55^{\circ}$ to $54^{\circ}$ and the peak frequency of the loss angle increases from approximately $154 \mathrm{~Hz}$ to $196 \mathrm{~Hz}$. In a word, as the number of orifices increases, the maximum dynamic stiffness and loss angle decrease slightly and the peak frequency of the loss angle increases significantly. Moreover, the HDB with more orifices can provide large damping in a wide frequency range. Therefore, the influences of the number of orifices on the performances of the HDBs are remarkable.

\section{Conclusions}

Hydraulically damped bushing (HDB) is crucial for the handling and NVH performances of vehicle. In this study, the dynamic stiffness and the loss angle of the HDBs with multiple internal configurations, which are the essential characteristics of the HDBs, are tested and the influences of 


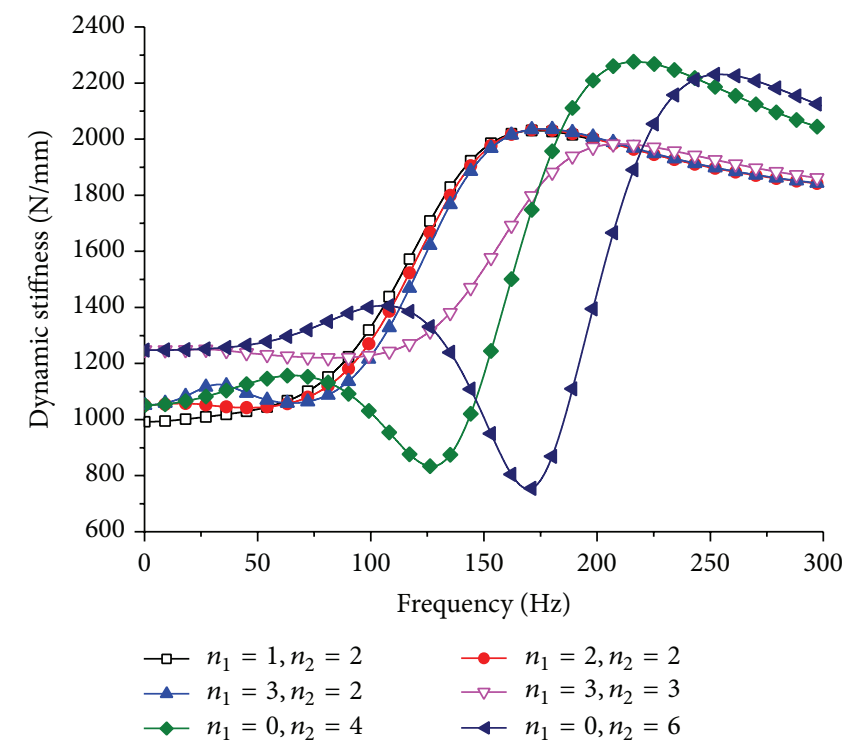

(a)

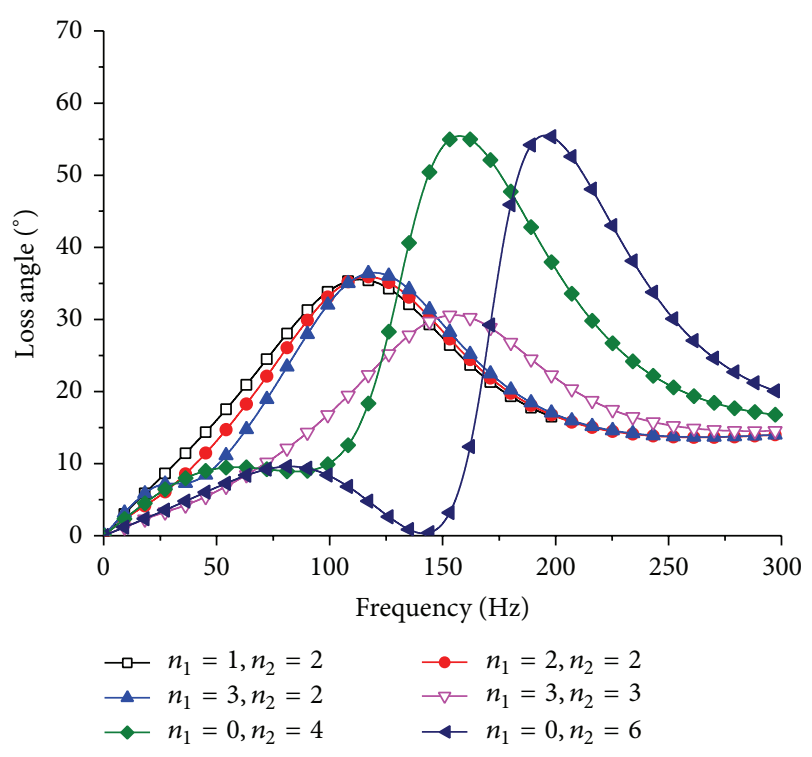

(b)

Figure 13: Calculated results of the dynamic characteristics of HDB with multiple tracks. (a) Dynamic stiffness. (b) Loss angle.

the HDB's structural features, namely, the number of inertial tracks and the cross-sectional shape of the track, are analyzed experimentally. It is found that (1) inertia tracks can only provide damping in low frequency range and their damping provision is almost negligible in high frequency range and (2) fixed points can be observed in test data for all HDB samples under all excitation conditions.

Lumped parameter (LP) models for the HDB with multiple inertia tracks and orifices are proposed in this paper in order to understand the relations between the HDB's structure features and its dynamic performance analytically. The LP models are validated via benchmarking the simulated dynamic stiffness and loss angle against test data. Based on the LP models, it is found that increasing the number of the inertial tracks and/or orifices would benefit the HDB's performance a great deal, since the dynamic stiffness, the amplitude, and the corresponding frequency of the loss angle would be significantly improved. However, it poses a question like whether there is a limitation on HDB's performance improvement by increasing the number of the inertial tracks. This motivates a future research that aims at finding a compromise between the complexity in manufacturing and assembly induced by the increase of fluid track number, with the improvement in HDB's performance. The proposed methods of modeling and analysis can be utilized to characterize the performances of the HDB with multiple inertia tracks and orifices before prototype.

\section{Conflict of Interests}

The authors declare that there is no conflict of interests regarding the publication of this paper.

\section{Acknowledgments}

The authors gratefully acknowledge the financial support from the Natural Science Foundation of China (Project nos. 51305139; 51475171), the Chinese Universities Scientific Fund (Project no. 2013ZM0016), and the Natural Science Foundation of Hubei Province (Project no. 2015CFB402). Experiments are made possible by equipment of nonresonant elastomer test machine (MTS 831) grants from Ningbo Tuopu Vibration Isolation System Co., Ltd., China.

\section{References}

[1] M. Lu and J. Ari-Gur, "Study of dynamic properties of automotive hydrobushing," ASME Design Engineering Division Proceedings, vol. 106, pp. 135-140, 2000.

[2] M. Lu and J. Ari-Gur, "Study of hydromount and hydrobushing with multiple inertia tracks," JSAE Annual Congress Proceedings, vol. 68, no. 2, pp. 5-8, 2002.

[3] W. Sauer and Y. Guy, "Hydro bushings-innovative NVH solutions in chassis technology," SAE Paper 2003-01-1475, 2003.

[4] T. Chai, R. Singh, and J. Dreyer, "Dynamic stiffness of hydraulic bushing with multiple internal configurations," SAE Paper 201301-1924, SAE International, 2013.

[5] T. Chai, J. Dreyer, and R. Singh, "Transient response of hydraulic bushing with inertia track and orifice-like elements," SAE Paper 2013-01-1927, 2013.

[6] T. Chai, J. T. Dreyer, and R. Singh, "Time domain responses of hydraulic bushing with two flow passages," Journal of Sound and Vibration, vol. 333, no. 3, pp. 693-710, 2014.

[7] T. Chai, J. T. Dreyer, and R. Singh, "Nonlinear dynamic properties of hydraulic suspension bushing with emphasis on the flow passage characteristics," Proceedings of the Institution of Mechanical Engineers, Part D: Journal of Automobile Engineering, vol. 229, no. 10, pp. 1327-1344, 2014. 
[8] T. Chai, J. T. Dreyer, and R. Singh, "Frequency domain properties of hydraulic bushing with long and short passages: system identification using theory and experiment," Mechanical Systems and Signal Processing, vol. 56-57, pp. 92-108, 2015.

[9] W. B. Shangguan and C. Xu, "Experiment and calculation methods of the dynamic performances for hydraulic bushings used in control arms of a suspension," Journal of Vibration and Shock, vol. 26, no. 9, pp. 7-10, 2007.

[10] Y.-Q. Zhang and W.-B. Shangguan, "A novel approach for lower frequency performance design of hydraulic engine mounts," Computers \& Structures, vol. 84, no. 8-9, pp. 572-584, 2006.

[11] B. Heißing and M. Ersoy, Chassis Handbook, Vieweg+Teubner, 2011.

[12] K. Seto, Dynamic Vibration Absorber and Its Applications, China Machine Press, Beijing, China, 2013.

[13] B. Piquet, C. A. Maas, and F. Capou, "Next generation of suspension bushings: review of current technologies and expansion upon new 3rd generation product data," SAE Paper 2007-010850, 2007.

[14] N. E. Lee, "Fluid damped resilient mounting," Patent 2582998, USA, 1952.

[15] G. L. Hipsher, "High damping resilient bushing," Article ID 364226, Patent 364226 8, USA, 1972.

[16] K. Konishi, "Fluid-filled resilient bushing," Patent 4588174 , USA, 1986.

[17] R. Kanda, "Fluid-filled resilient bushing with circumferential orifice," Patent 4693456, USA, 1987.

[18] R. Kanda, "Fluid-filled cylindrical elastic connector having two orifice passages with different cross sectional areas," Patent 5060918, USA, 1991.

[19] A. Vossel and F. Meyerink, "Hydraulically damping rubber bearing with decoupling element," Patent 6511058, USA, 2003.

[20] F. Meyerink and C. Hoping, "Hydro-bush bearing with acoustic decoupling," Patent Application 11/760846, USA, 2007.

[21] M. Sevensson and M. Hakansson, Hydrobushing model for multi-body simulation [M.S. thesis], Lund University, Lund, Sweden, 2004.

[22] X.-Y. Pan, X.-X. Xie, and W.-B. Shangguan, "Dynamic properties analysis for hydraulic rubber isolator under excitations with different amplitudes," Chinese Journal of Vibration and Shock, vol. 31, no. 1, pp. 144-149, 2012.

[23] S. Arzanpour and F. Golnaraghi, "A novel semi-active magnetorheological bushing design for variable displacement engines," Journal of Intelligent Material Systems and Structures, vol. 19, no. 9, pp. 989-1003, 2008.

[24] S. Arzanpour and M. F. Golnaraghi, "Development of an active compliance chamber to enhance the performance of hydraulic bushings," Journal of Vibration and Acoustics-Transactions of the ASME, vol. 132, no. 4, pp. 1-7, 2010.

[25] R. Singh, G. Kim, and P. V. Ravindra, "Linear analysis of automotive hydro-mechanical mount with emphasis on decoupler characteristics," Journal of Sound and Vibration, vol. 158, no. 2, pp. 219-243, 1992.

[26] W.-B. Shangguan and Z.-H. Lu, "Experimental study and simulation of a hydraulic engine mount with fully coupled fluidstructure interaction finite element analysis model," Computers \& Structures, vol. 82, no. 22, pp. 1751-1771, 2004.

[27] G. Kim and R. Singh, "A study of passive and adaptive hydraulic engine mount systems with emphasis on non-linear characteristics," Journal of Sound and Vibration, vol. 179, no. 3, pp. 427-453, 1995.
[28] R. Fan and Z. Lu, "Fixed points on the nonlinear dynamic properties of hydraulic engine mounts and parameter identification method: experiment and theory," Journal of Sound and Vibration, vol. 305, no. 4-5, pp. 703-727, 2007.

[29] L.-R. Wang, Z.-H. Lu, and I. Hagiwara, "Analytical analysis approach to nonlinear dynamic characteristics of hydraulically damped rubber mount for vehicle engine," Nonlinear Dynamics, vol. 61, no. 1-2, pp. 251-264, 2010.

[30] J. Z. Lin, X. D. Ruan, B. G. Chen et al., Fluid Mechanics, Tsinghua University Press, Beijing, China, 2010.

[31] J.-H. Lee and K.-J. Kim, "An efficient technique for design of hydraulic engine mount via design variable-embedded damping modeling," Journal of Vibration and Acoustics, vol. 127, no. 1, pp. 93-99, 2005.

[32] B. Barszcz, J. T. Dreyer, and R. Singh, "Experimental study of hydraulic engine mounts using multiple inertia tracks and orifices: narrow and broad band tuning concepts," Journal of Sound and Vibration, vol. 331, no. 24, pp. 5209-5223, 2012.

[33] E. Doebelin, System Dynamics: Modeling, Analysis, Simulation, Design, CRC Press, New York, NY, USA, 1998. 


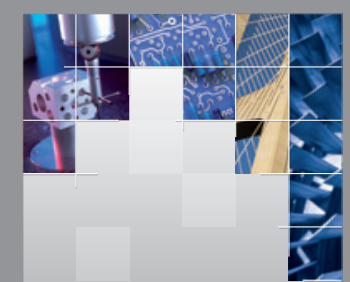

\section{Enfincering}
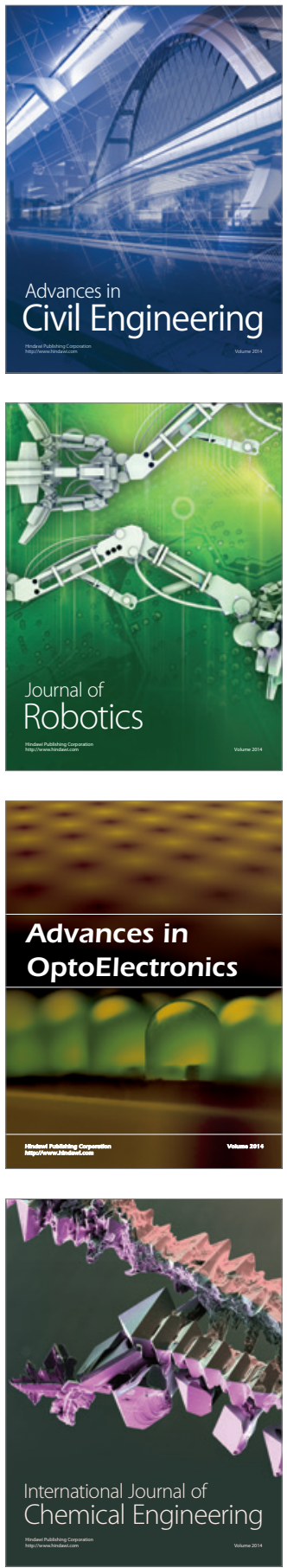

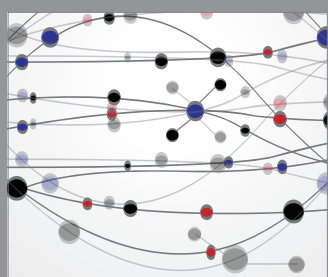

The Scientific World Journal

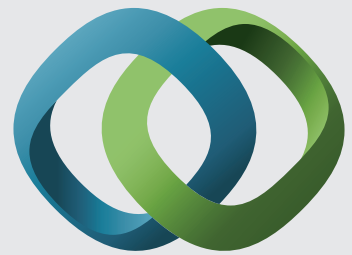

\section{Hindawi}

Submit your manuscripts at

http://www.hindawi.com
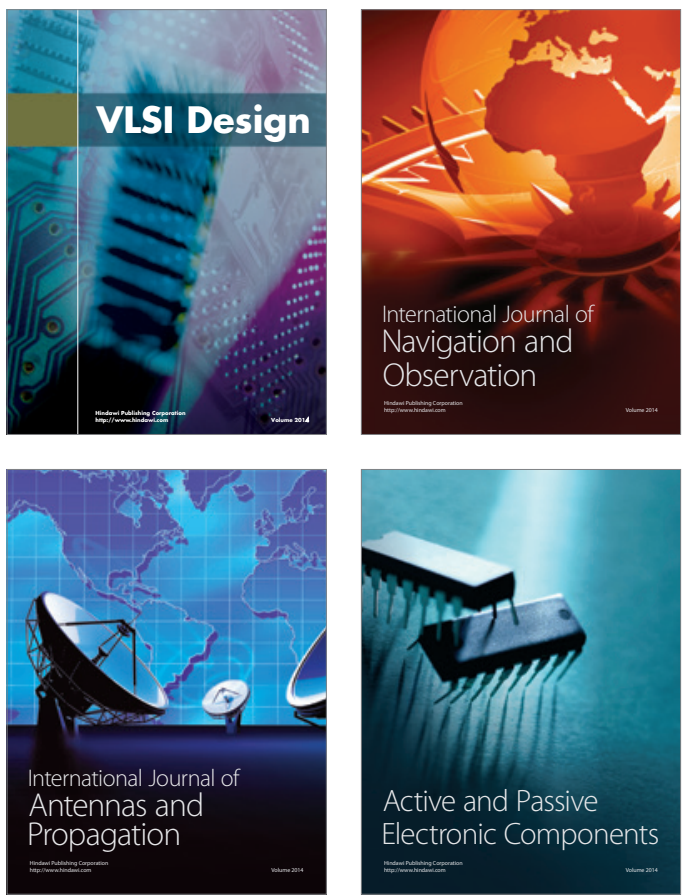
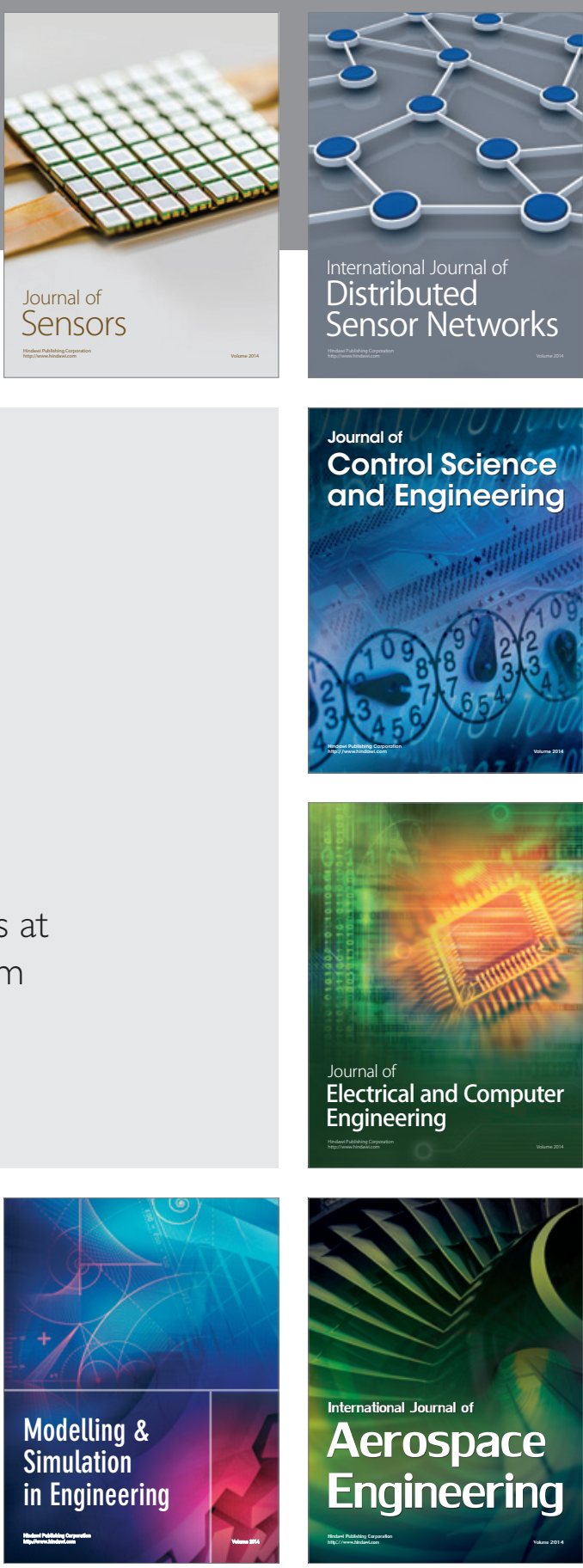

International Journal of

Distributed

Sensor Networks

Journal of

Control Science

and Engineering
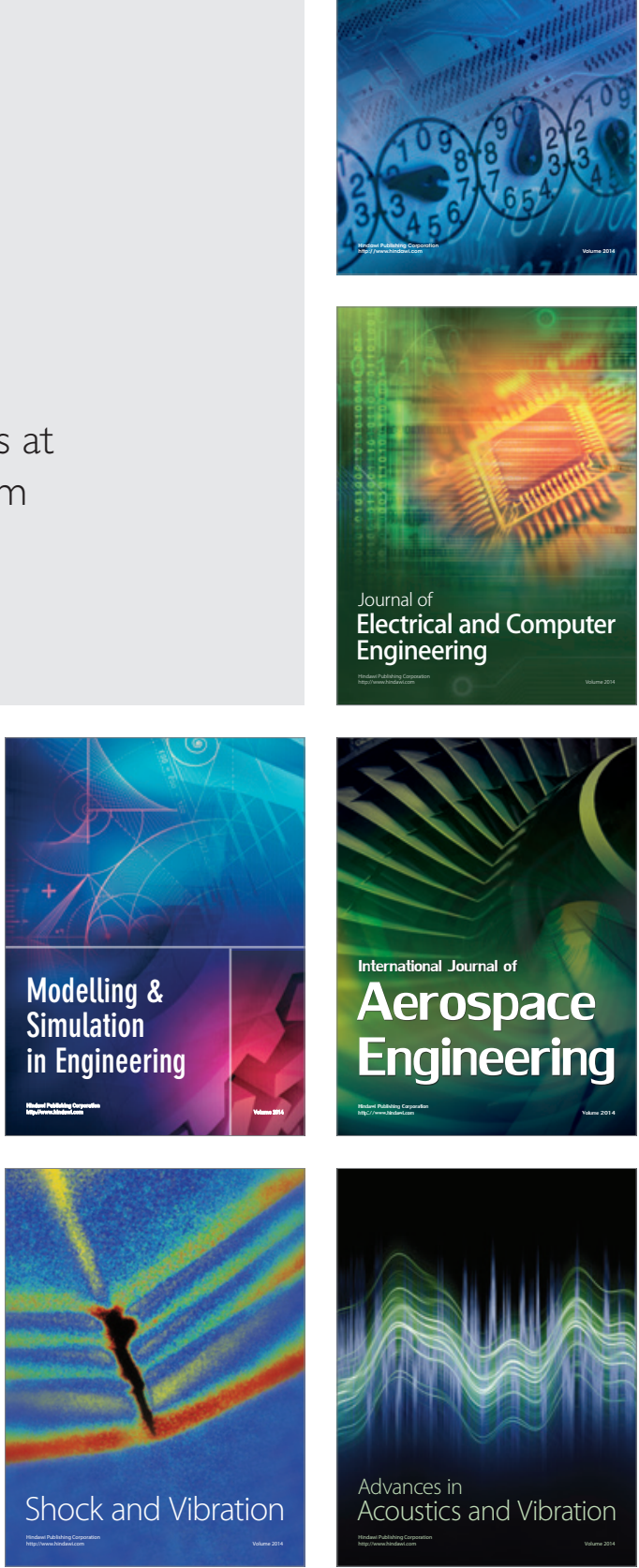